Nonlinear time series analysis of jerk congenital nystagmus

Akman, O. E. and Broomhead, D. S. and Clement, R. A. and Abadi, R. V.

2006

MIMS EPrint: 2006.27

Manchester Institute for Mathematical Sciences

School of Mathematics

The University of Manchester

\footnotetext{
Reports available from: http://eprints.maths.manchester.ac.uk/

And by contacting: The MIMS Secretary

School of Mathematics

The University of Manchester

Manchester, M13 9PL, UK
} 


\title{
Nonlinear time series analysis of jerk congenital nystagmus.
}

\author{
O.E. Akman* \\ The School of Mathematics, The University of Manchester \\ P.O. Box 88, Manchester M60 1QD, U.K. \\ D.S. Broomhead \\ The School of Mathematics, The University of Manchester \\ P.O. Box 88, Manchester M60 1QD, U.K. \\ R.A. Clement \\ Visual Sciences Unit, Institute of Child Health, U.C.L. \\ 30 Guilford Street, London WC1N 1EH, U.K. \\ R.V. Abadi \\ Faculty of Life Sciences, Moffat Building, The University of Manchester \\ Sackville St, Manchester M60 1QD, U.K.
}

\begin{abstract}
Nonlinear dynamics provides a complementary framework to control theory for the quantitative analysis of the oculomotor control system. This paper presents a number of findings relating to the aetiology and mechanics of the pathological ocular oscillation jerk congenital nystagmus (jerk $\mathrm{CN}$ ). A range of time series analysis techniques were applied to both recorded jerk $\mathrm{CN}$ waveforms and simulated waveforms produced by an established model in which the oscillations are a consequence of an unstable neural integrator. The results of the time series analysis were then interpreted within the framework of a generalised model of the unforced oculomotor system.

This work suggests that for jerk oscillations, the origin of the instability lies in one of the five oculomotor subsystems, rather than in the final common pathway (the neural integrator and muscle plant). Additionally, experimental estimates of the linearised foveation dynamics imply that a refixating fast phase induced by a near-homoclinic trajectory will result in periodic oscillations. Local dimension calculations show that the dimension of the experimental jerk $\mathrm{CN}$ data increases during the fast phase, indicating that the oscillations are not periodic, and hence that the refixation mechanism is of greater complexity than a homoclinic reinjection. The dimension increase is hypothesised to result either from a signal-dependent noise process in the saccadic system, or the activation of additional oculomotor components at the beginning of the fast phase. The modification of a recent saccadic system model to incorporate biologically realistic signal-dependent noise is suggested, in order to test the first of these hypotheses.
\end{abstract}

*Now at: Mathematics Institute, University of Warwick, Coventry CV4 7AL, UK 
The oculomotor system is well suited to the quantitative study of sensorimotor processing (Robinson, 1986). Single cell recordings have enabled the identification of many of the neural pathways associated with eye movement control, enabling meaningful mathematical models of the control circuitry to be developed. Additionally, eye movements can be accurately recorded in the laboratory, providing high quality time series data which can be used to test and develop such models.

The majority of models developed thus far have been based on control theory (Carpenter, 1988). These models have had considerable success in elucidating the functional organisation of the oculomotor system (Leigh and Zee, 1999). A significant achievement of the control approach has been the prediction of a common neural integrator, subsequently confirmed experimentally (Robinson, 1968; Cohen and Komatsuzaki, 1972; Zee et al., 1981; Cannon and Robinson, 1987). This successful prediction supported the assumption that individual blocks of the control models can be directly identified with separate classes of neurons in the brain stem. However, in subsequent modelling of pathological eye movements (Optican and Zee, 1984; Jacobs and Dell'Osso, 2004), the number of assumed blocks far exceeds the number of known classes of neurons so that this assumption becomes questionable, motivating the need for alternative modelling strategies.

Techniques based on nonlinear dynamics provide a complementary approach to control theory in the analysis of the oculomotor system (Clement et al., 2002a). The last few years have seen the development of differential equation models (Gancarz and Grossberg, 1998; Broomhead et al., 2000) together with the application of time series analysis to eye movement recordings (Shelhamer, 1997; Abadi et al., 1997; Clement et al., 2002b,c). A previous paper showed that one advantage of the nonlinear dynamics approach is the capacity of bifurcation analysis to reveal the full range of behaviour that a model is capable of producing (Akman et al., 2005). The present paper describes the use of nonlinear time series techniques to investigate hypotheses suggested by oculomotor models regarding the aetiology and mathematical characterisation of oscillatory ocular disorders. More generally, it is demonstrated that these techniques provide a quantitative means of testing such models against experimental data.

Section 2 of the paper introduces the reader to the functional organisation of the different oculomotor subsystems. Thereafter, section 3 details the construction of a generalised model of the unforced oculomotor system for horizontal eye movements. This model provides a framework for interpreting the results of the time series analysis. The characterisation of a pathological oculomotor system in terms of the model is discussed in section 4, where it is hypothesised that the condition known as jerk congenital nystagmus (jerk $\mathrm{CN}$ ) is a consequence of a bifurcation. Section 5 describes the data reconstruction method (delay embedding) which forms the basis of the computational techniques used to check and expand upon this hypothesis. The results of applying these techniques to both 
recorded jerk $\mathrm{CN}$ data and waveforms simulated by an unstable neural integrator model are presented in section 6 . The implications of the work are discussed in section 7 .

The results of this study indicate that in jerk CN, the initial instability is caused by a fixed point (that corresponds to stable gaze at the primary position, $0^{\circ}$ ) losing stability in a 1-dimensional bifurcation. Moreover, estimates of the eigenvalues of the linearisation at this fixed point suggest that the most likely source of this bifurcation is one of the oculomotor subsystems, rather than the neural integrator or muscle plant. Additionally, a model of the linearised dynamics about the fixed point derived from the eigenvalue estimates implies that a fast phase comprising a deterministic refixation induced by a near-homoclinic orbit will result in a limit cycle attractor. Local dimension calculations show that the dimension of the jerk $\mathrm{CN}$ attractor increases during the fast phase, implying that a limit cycle is not a reasonable approximation to the true behaviour. Two possible mechanisms that could account for the dimension increase are discussed: the activation of additional oculomotor components as the cycle enters the fast phase, or a signal-dependent increase in the variance of the saccadic control signal. It is remarked that both would result in a fast phase that is dynamically more complex than a homoclinic reinjection.

More generally, it is suggested that the eigenvalues of linearisation and local dimension are quantitative experimental measurements that can be used in the development of comprehensive CN models. Within this framework, it is proposed that a model capable of simulating the local dimension variation could be obtained by modifying an existing saccadic system model to incorporate realistic signal-dependent noise.

\section{Oculomotor control and congenital nystagmus}

The oculomotor system controls the movement of the eyes so as to ensure that the image of the object of interest falls on the high resolution region of the retina called the fovea. This process is referred to as foveation (Ditchburn, 1973). Resolution of detail decreases sharply away from the fovea, and is also degraded if images slip over the fovea at velocities greater than a few degrees per second. Optimal visual performance is therefore only attained when images are held steady on this region (Westheimer and McKee, 1975).

Depending on the stimuli and viewing conditions, the foveation task can involve up to five oculomotor subsystems: the saccadic, smooth pursuit, vestibular, optokinetic and vergence systems. The saccadic system provides rapid shifts of gaze (saccades) to bring about foveation of new targets. The smooth pursuit system matches eye velocity with target velocity to provide a stable foveal image when tracking slow-moving objects in the visual field. The function of the vestibular system is to stabilise gaze during brief head rotations by generating an eye movement which has velocity equal and opposite to head velocity. The optokinetic system matches eye velocity to the global retinal image velocity in order to maintain stable gaze during sustained head motion. Finally, the vergence system 
maintains foveation during motion of the target towards or away from the eyes (Carpenter, 1988; Leigh and Zee, 1999).

The vestibular system is driven by non-visual signals from the semicircular canals, while the other systems are driven by visual signals encoding target information. In response to this sensory input, each of the oculomotor subsystems generates a velocity-coded command signal. A copy of this signal is sent to a complex of neurons, the common neural integrator (NI), which integrates it to produce a position-coded signal. The position and velocity signals are then summed and relayed to the relevant ocular motoneurons. These send the final motor command to the eye muscles, producing a shift in the eye position (Carpenter, 1988; Leigh and Zee, 1999). In control models, the ocular motoneurons and eye muscles are referred to collectively as the muscle plant. Here, the five oculomotor subsystems will be referred to as the oculomotor command system (OCS). A schematic representation of the oculomotor system architecture is shown in figure 1.

Congenital nystagmus $(\mathrm{CN})$ is an involuntary, bilateral oscillation of the eyes that is present in approximately 1 in 4000 of the population (Abadi and Dickinson, 1986; Abadi and Bjerre, 2002). The oscillations are conjugate and occur primarily in the horizontal plane. The oscillation generally consists of a slow phase, which takes the retinal image of the visual target away from the fovea, followed by a fast or slow phase which moves the image back onto the fovea (Dell'Osso and Daroff, 1975; Abadi and Dickinson, 1986; Abadi et al., 1991). CN subjects tend to have poor visual acuity due to the reduced foveation time (Abadi and Sandikcioglu, 1975; Abadi and Worfolk, 1989; Bedell and Loshin, 1991). A common waveform observed in adults is jerk $\mathrm{CN}$, composed of an increasing exponential slow phase followed by a saccadic fast phase. The oscillation is referred to as right-beating or left-beating depending on the direction of the fast phase. Recordings from two subjects with left-beating jerk nystagmus can be seen in figure 2 .

\section{Modelling the oculomotor control system for horizontal eye movements: a nonlinear dynamics approach}

Quantitative investigations of the dynamics of the muscle plant have revealed that it can be modelled as a linear system which is at least second order (Robinson, 1964; Goldstein, 1987). Writing $x^{(j)}$ for the $j$ th time derivative of $x$, the most general equation describing the plant dynamics is thus

$$
\begin{array}{r}
g^{(k)}+p_{k-1} g^{(k-1)}+\ldots+p_{1} \dot{g}+p_{0} g=q_{l}\left(R n^{(l)}+b^{(l)}\right)+q_{l-1}\left(R n^{(l-1)}+b^{(l-1)}\right) \\
+\ldots+q_{1}(R \dot{n}+\dot{b})+q_{0}(R n+b)
\end{array}
$$

with $k \geq 2, l \geq 0$ and $q_{0} \neq 0$. Here, $g$ is horizontal eye position, $n$ is the NI output, $b=\sum_{K=S, P, V, O K N, V G} b_{K}$ is the signal from the OCS (cf. figure 1) and $R$ is a positive 
constant which determines the relative weighting of the position and velocity inputs to the muscle plant.

The equation for the NI output $n$ is

$$
\dot{n}=-\frac{1}{N_{T}} n+b
$$

where the time constant $N_{T}$ determines the drift of the eye back to primary position from eccentric gaze. $N_{T}$ is of the order of $25 \mathrm{~s}$ in normal subjects (Becker and Klein, 1973). When it can be assumed that the sensory input to the oculomotor subsystems is negligible (such as during viewing of a stationary target in the primary position, or when the eyes are closed), the dynamics of each oculomotor subsystem can be approximated by a set of autonomous, ordinary differential equations. The combined dynamics of the unforced OCS can thus be written as $\dot{\mathbf{y}}=\mathbf{f}(\mathbf{y})$, where $\mathbf{y} \in \mathbb{R}^{q}$ (with $q$ a finite integer) combines the state vectors of each subsystem, and $\mathbf{f}$ combines the corresponding vector fields. The velocity command $b$ can thus be expressed as $b=G(\mathbf{y})$ for some scalar function $G$, where $G$ incorporates any velocity biases that may result in a nonzero neural integrator null position (Goldman et al., 2001).

Introducing the state vector $\mathbf{x}=\left(g, \dot{g}, \ldots, g^{(k-1)}\right)^{T}$ enables the plant equation (1) to be written in the vectorised form

$$
\dot{\mathbf{x}}=A \mathbf{x}+\mathbf{S}(n, \mathbf{y})
$$

where $A$ is the $k \times k$ matrix

$$
A=\left(\begin{array}{ccccc}
0 & 1 & 0 & \ldots & 0 \\
0 & 0 & 1 & \ldots & 0 \\
\vdots & \vdots & \vdots & \ddots & \vdots \\
0 & 0 & 0 & \ldots & 1 \\
-p_{0} & -p_{1} & -p_{2} & \ldots & -p_{k-1}
\end{array}\right)
$$

and $\mathbf{S}(n, \mathbf{y})=(0, \ldots, 0, S(n, \mathbf{y}))^{T} \in \mathbb{R}^{k}$, with $S(n, \mathbf{y})$ a scalar function representing the right-hand-side of (1) expressed as a function of $n$ and $\mathbf{y}$. The final equations for the unforced oculomotor dynamics are thus

$$
\begin{aligned}
\dot{\mathbf{x}} & =A \mathbf{x}+\mathbf{S}(n, \mathbf{y}) \\
\dot{n} & =-\frac{1}{N_{T}} n+G(\mathbf{y}) \\
\dot{\mathbf{y}} & =\mathbf{f}(\mathbf{y}) .
\end{aligned}
$$

These will sometimes be written in the condensed form $\dot{\mathbf{z}}=\mathbf{F}(\mathbf{z})$ for convenience, where $\mathbf{z}=(\mathbf{x}, n, \mathbf{y}) \in \mathbb{R}^{D}$, with $D=k+q+1$. The equations have a skew-product form, as the vector field of (4) does not contain any terms in $\mathbf{x}$ and $n$. The plant and neural integrator dynamics (2)-(3) are thus slaved to the OCS dynamics (4), which determine the qualitative behaviour of the full system $\dot{\mathbf{z}}=\mathbf{F}$ (z) (Stark, 1999). 


\section{Characterisation of a pathological oculomotor system}

In terms of the modelling approach presented here, a normal oculomotor system corresponds to the unforced dynamics $\dot{\mathbf{z}}=\mathbf{F}(\mathbf{z})$ having a single, stable fixed point $\overline{\mathbf{z}}$ of the form $\overline{\mathbf{z}}=(0, \ldots 0, \bar{n}, \overline{\mathbf{y}})$. For such a system, after motion of the eye driven by sensory input, the eye comes to rest at the primary position. A pathological oculomotor system - such as jerk $\mathrm{CN}$ - develops when $\overline{\mathbf{z}}$ becomes unstable through a bifurcation, leading to an attractor for which the eye does not come to rest at $0^{\circ}$ (e.g. a stable fixed point with $g \neq 0$, a stable limit cycle, a strange attractor). ${ }^{1}$

Equations (2)-(4) imply that the Jacobian derivative $D_{\mathbf{z}} \mathbf{F}(\overline{\mathbf{z}})$ of $\mathbf{F}(\mathbf{z})$ at $\overline{\mathbf{z}}$ is given by

$$
D_{\mathbf{z}} \mathbf{F}(\overline{\mathbf{z}})=\left(\begin{array}{ccc}
A & \frac{\partial \mathbf{S}}{\partial n}(\bar{n}, \overline{\mathbf{y}}) & \frac{\partial \mathbf{S}}{\partial \mathbf{y}}(\bar{n}, \overline{\mathbf{y}}) \\
\mathbf{0}_{1 \times k} & -\frac{1}{N_{T}} & \frac{\partial G}{\partial \mathbf{y}}(\overline{\mathbf{y}}) \\
\mathbf{0}_{q \times k} & \mathbf{0}_{q \times 1} & D_{\mathbf{y}} \mathbf{f}(\overline{\mathbf{y}})
\end{array}\right)
$$

where $\mathbf{0}_{r \times s}$ is the $r \times s$ matrix of zeros. Writing $-\frac{1}{T_{j}}$ for the $j$ th eigenvalue ${ }^{2}$ of $A$, it follows from the block upper-triangular form of $D_{\mathbf{z}} \mathbf{F}(\overline{\mathbf{z}})$ that its eigenvalues are $\left\{-\frac{1}{T_{1}},-\frac{1}{T_{2}}, \ldots,-\frac{1}{T_{k}}\right\}$ together with $-\frac{1}{N_{T}}$ and the eigenvalues of $D_{\mathbf{y}} \mathbf{f}(\overline{\mathbf{y}}) \cdot{ }^{3}$ Since $T_{j}>0$ for $1 \leq j \leq k, \overline{\mathbf{z}}$ can only be unstable if the neural integrator is unstable $\left(N_{T}<0\right)$ or $\overline{\mathbf{y}}$ is an unstable fixed point of the OCS $\dot{\mathbf{y}}=\mathbf{f}(\mathbf{y})$ (i.e. $D_{\mathbf{y}} \mathbf{f}(\overline{\mathbf{y}})$ has one or more eigenvalues with positive real part). Of these two possibilities, Optican et al suggested the former as a mechanism for generating jerk CN, on the basis of a control model (Optican and Zee, 1984). In their model, the slow phases of the waveform are a consequence of an unstable NI, resulting from a pathological positive velocity feedback loop. The fast phases are corrective saccades, triggered when the eye position exceeds a threshold level (Optican and Zee, 1984). In terms of the present notation, the unstable NI model posits that $\overline{\mathbf{z}}$ has lost stability as a result of the time constant $N_{T}$ changing sign, giving a positive eigenvalue of $D_{\mathbf{z}} \mathbf{F}(\overline{\mathbf{z}})$, rather than the eigenvalue -0.04 characteristic of normal subjects. The alternative - that $\overline{\mathbf{y}}$ undergoes a bifurcation in the OCS $\dot{\mathbf{y}}=\mathbf{f}(\mathbf{y})$ - was suggested in (Broomhead et al., 2000), where an instability of the saccadic system was considered. In both cases, it can be proved that the skew-product form of $\dot{\mathbf{z}}=\mathbf{F}(\mathbf{z})$ implies that the corresponding bifurcation in the full system will be of the same type (for example, a Hopf bifurcation in the OCS will induce a Hopf bifurcation in the full system).

\footnotetext{
${ }^{1}$ The centripetal oscillations observed in some normal subjects as they fixate a target at an eccentric gaze angle (physiological endpoint nystagmus) do not correspond to a pathological system by this definition, since they are driven by visual signals, rather than an endogenous instability; once the subject stops attempting to maintain the eccentric eye position, the oscillations cease.

${ }^{2}$ Conventionally, it is assumed that the plant is overdamped (Robinson, 1964; Optican and Zee, 1984; Goldstein, 1987).

${ }^{3}$ It should be noted that the block upper-triangular form of $D_{\mathbf{z}} \mathbf{F}(\overline{\mathbf{z}})$ is a consequence of $\dot{\mathbf{z}}=\mathbf{F}(\mathbf{z})$ being a skew-product.
} 


\section{Delay embedding of jerk CN waveforms}

In order to test the hypothesis that jerk $\mathrm{CN}$ is caused by the bifurcation of a fixed point $\overline{\mathbf{z}}$ corresponding to stable fixation at the primary position, a number of methods from nonlinear time series analysis were applied to data obtained from two adult subjects (A and B) with jerk nystagmus. Both subjects had conjugate and uniplanar eye movements, with Snellen visual acuities of 6/12 in the right and left eyes. Neither subject exhibited any ocular or neurological disorders. Informed consent was obtained according to the Declaration of Helsinki.

Binocular horizontal eye movements were monitored using an IRIS 6500 (Skalar Medical) system, a head mounted device based on the infrared reflection method. The head was restrained by a head rest and the subjects instructed to fixate a $1^{\circ}$ stationary distant target in the primary position for 30s. Calibration was carried out by asking the subjects to follow a sinusoidally moving stimulus with an amplitude of $\pm 5^{\circ}$. The analogue output of the system was filtered through a $100 \mathrm{~Hz}$ low-pass filter, sampled at $5 \mathrm{~ms}$ intervals for $10 \mathrm{~s}$ and then digitised to 12 bit accuracy. The system was linear to $\pm 20^{\circ}$ with a resolution of $0.03^{\circ}$. For both subjects, the time series obtained from the left eye was used for the analysis. Blinks were removed by thresholding an estimate of eye velocity obtained by convolving the traces with an 11 point derivative filter (Gibson et al., 1992). Figure 2 shows portions of the time series recorded from the two subjects.

The same computational techniques applied to the recorded CN traces were also applied to a simulated jerk waveform generated by the unstable NI model of Optican et al ((Optican and Zee, 1984)), to determine the extent to which such a model is able to account for the experimental data. In order to mimic the observational noise produced by the IRIS 6500 , the simulated time series was sampled at $5 \mathrm{~ms}$ intervals for $10 \mathrm{~s}$, and Gaussian white noise with standard deviation equal to the system resolution was added to the sampled signal. ${ }^{4}$ Portions of the resulting time series can be seen in the right panel of figure 7 .

\subsection{The method of delays}

The techniques used here are based on the method of delays (Broomhead and King, 1986; Gibson et al., 1992; Ott et al., 1994; Kantz and Schreiber, 1997). As stated above, eye movements were recorded from the $\mathrm{CN}$ subjects during viewing of a stationary target in the primary position. It can therefore be assumed that these time series have been obtained by sampling the eye position component $g(\mathbf{z}(t))$ of a particular trajectory $\left\{\mathbf{z}(t): t \geq 0, \mathbf{z}(0)=\mathbf{z}_{0}\right\}$ of $\dot{\mathbf{z}}=\mathbf{F}(\mathbf{z})$ every $\tau$ seconds $(\tau=0.005)$ a total of $N$ times $(N \leq 2000)$. Let $\mathbf{z}_{k}=\mathbf{z}(k \tau)$ and $g_{k}=g\left(\mathbf{z}_{k}\right)$ for $1 \leq k \leq N$, so that $\left\{\mathbf{z}_{1}, \mathbf{z}_{2}, \ldots, \mathbf{z}_{N}\right\}$ is the

\footnotetext{
${ }^{4}$ As the quantisation error associated with the analogue-to-digital conversion process is much smaller than $0.03^{\circ}$, the dominant source of observational error introduced by the IRIS 6500 is due to the resolution constraints of the device.
} 
sampled trajectory and $\left\{g_{1}, g_{2}, \ldots, g_{N}\right\}$ is the corresponding time series. The delay vectors $\left\{\mathbf{w}_{1}, \mathbf{w}_{2}, \ldots, \mathbf{w}_{N-d+1}\right\}$ obtained by taking delays of length $d$ are then defined as follows:

$$
\mathbf{w}_{k}=\left(g_{k}, g_{k+1}, \ldots, g_{k+d-1}\right)^{T} .
$$

It is also assumed that asymptotically, the dynamics of $\dot{\mathbf{z}}=\mathbf{F}(\mathbf{z})$ are confined to an attractor $\mathcal{A}$ lying in an $m$-dimensional, smooth, compact manifold with boundary $M \subset \mathbb{R}^{D}$. Letting $\left\{\phi_{t}: t \in \mathbb{R}\right\}$ represent the flow of $\dot{\mathbf{z}}=\mathbf{F}(\mathbf{z})$, so that $\mathbf{z}(t)=\phi_{t}\left(\mathbf{z}_{0}\right), \mathbf{w}_{k}$ can be written as $\mathbf{w}_{k}=\boldsymbol{\Phi}\left(\mathbf{z}_{k}\right)$ where the delay map $\boldsymbol{\Phi}: M \rightarrow \mathbb{R}^{d}$ is defined by

$$
\mathbf{\Phi}(\mathbf{z})=\left(g(\mathbf{z}), g\left(\phi_{\tau}(\mathbf{z})\right), \ldots, g\left(\phi_{(d-1) \tau}(\mathbf{z})\right)\right)^{T} .
$$

A well-known result from applied dynamical systems theory, due to Takens, implies that if $d \geq 2 m+1$ and certain genericity conditions hold, $\boldsymbol{\Phi}$ is an embedding of $M$ in $\mathbb{R}^{d}$; i.e. $\boldsymbol{\Phi}(M)$ is a smooth submanifold of $\mathbb{R}^{d}$ with $\mathbf{\Phi}: M \rightarrow \boldsymbol{\Phi}(M)$ a diffeomorphism (a smooth map with a smooth inverse) (Takens, 1981). ${ }^{5}$ The delay map therefore induces a flow $\psi_{t}=\boldsymbol{\Phi} \circ \phi_{t} \circ \boldsymbol{\Phi}^{-1}$ on $\boldsymbol{\Phi}(M)$ such that the dynamics on $\mathbf{\Phi}(M)$ under $\psi_{t}$ are equivalent to the dynamics on $M$ under $\phi_{t}$, up to the smooth change of coordinates $\boldsymbol{\Phi}$. The dynamics on $M$ are thus reconstructed in $\mathbb{R}^{d}$ by the delay map. Indeed, many important quantities defined by $\phi_{t}$ are invariant under the embedding. These are coordinate-independent quantities, such as the dimensions of $\mathcal{A}$ and $M$, the Lyapunov exponents of the flow and the eigenvalues of linearisation at fixed points. Moreover, because $\mathbf{w}_{k+1}=\psi_{\tau}\left(\mathbf{w}_{k}\right)$, the sequence $\left\{\mathbf{w}_{1}, \mathbf{w}_{2}, \ldots, \mathbf{w}_{N-d+1}\right\}$ is a sampled trajectory of the reconstructed dynamics, and hence the invariant quantities can be recovered from the delay vectors (Broomhead and King, 1986; Healey et al., 1991; Gibson et al., 1992; Ott et al., 1994; Kantz and Schreiber, 1997).

\subsection{Choice of the delay length $d$}

A useful tool when determining a suitable choice of the delay length $d$ is the global trajectory matrix $X$ of the time series. $X$ is defined as the $(N-d+1) \times d$ matrix whose $k$ th row is $\frac{1}{\sqrt{N}} \mathbf{w}_{k}^{T}$. The singular value decomposition (SVD) of $X$ provides information on the distribution of the delay vectors in $\mathbb{R}^{d}$ (Broomhead and King, 1985). Assuming that the time series is stationary with zero mean, the right singular vectors of $X$ provide an orthonormal basis of $\mathbb{R}^{d}$ such that in the limit $N \rightarrow \infty$, the coordinates of the delay vectors in the new basis are uncorrelated. Moreover, the variance of the $j$ th coordinate of the delay vectors in the SVD basis is $\sigma_{j}^{2}$, where $\sigma_{j}$ is the singular value of $X$ corresponding to the $j$ th right singular vector (Broomhead and King, 1986). By convention, $\sigma_{j} \geq \sigma_{j-1}$. Hence, if the first $d-1$ singular vectors of $X$ contain a significant proportion of the total variance

\footnotetext{
${ }^{5}$ The original Takens' Theorem assumes that $M$ is a smooth, compact submanifold of the system's phase space. For Euclidian phase spaces, however, it is often the case that the attractor lies in a compact ball, that is, a smooth, compact manifold with boundary. (Huke, 1993) extends Takens Theorem to this case.
} 
(i.e. $\sum_{j=1}^{d-1} \sigma_{j}^{2} / \sum_{j=1}^{d} \sigma_{j}^{2} \approx 1$ ), it can be inferred that the image of $\mathcal{A}$ under the delay map $\boldsymbol{\Phi}$ lies in a $d-1$ dimensional linear subspace of $\mathbb{R}^{d}$, and hence that $M$ has effectively been reconstructed in $\mathbb{R}^{d}$ by $\boldsymbol{\Phi}$. (It should be noted that this does not necessarily imply that $\boldsymbol{\Phi}$ is an embedding, although this is a reasonable working assumption).

For the time series of subjects $\mathrm{A}$ and $\mathrm{B}, \sum_{j=1}^{d-1} \sigma_{j}^{2} / \sum_{j=1}^{d} \sigma_{j}^{2}>0.9999$ for $d \geq 7$ in both cases; i.e. for $d \geq 7$, more than $99.99 \%$ of the variance lies in the first $d-1$ singular vectors. In view of this, $d$ was taken equal to 7 in the subsequent analysis of both sets of data. Applying the same analysis to the simulated waveform from the unstable NI model, it was also found that $\sum_{j=1}^{d-1} \sigma_{j}^{2} / \sum_{j=1}^{d} \sigma_{j}^{2}>0.9999$ for $d \geq 7 ; d$ was therefore set to 7 in the analysis of this data set as well.

\section{Analysis of the reconstructed attractors}

This section presents the main results of the paper. Section 6.1 describes a technique for estimating fixed points of the reconstructed jerk CN dynamics, and shows the result of carrying out this procedure on the experimental and model time series. Sections 6.2 and 6.3 provide a theoretical background to two further techniques; the computation of the local dimension of the reconstructed attractor, and the estimation of eigenvalues of linearisation at fixed points of $\dot{\mathbf{z}}=\mathbf{F}(\mathbf{z})$ from the delay vectors. Section 6.4 gives the result of applying these techniques to the experimental and model data sets in the neighbourhood of the delay vectors obtained from the fixed point estimation process, and the subsequent inferences that can be made regarding the origin of the jerk instability.

Following this, section 6.5 details the construction of an analytical Poincaré map obtained by combining the eigenvalue estimates from the experimental data with a fast phase modelled as a homoclinic reinjection. An analysis of this map implies that the jerk CN attractor should be a limit cycle. Finally, section 6.6 describes the use of local dimension calculations at different points on the reconstructed data sets to determine whether a limit cycle is a reasonable approximation to the actual behaviour.

\subsection{Estimates of the reconstructed hypothetical fixed points}

If a fixed point $\overline{\mathbf{z}}$ of $\dot{\mathbf{z}}=\mathbf{F}(\mathbf{z})$ does exist, its image under $\mathbf{\Phi}$ is a fixed point of the dynamical system induced by $\boldsymbol{\Phi}$, and must lie in a region where the velocity of the flow is low. Writing $\overline{\mathbf{w}}=\boldsymbol{\Phi}(\overline{\mathbf{z}})$, the form of $\boldsymbol{\Phi}$ implies that $\overline{\mathbf{w}}$ would lie on the principal diagonal $\operatorname{Sp}\left((1, \ldots, 1)^{T}\right)$ of the delay space $\mathbb{R}^{d}$. A reasonable estimate of $\overline{\mathbf{w}}$ is therefore provided by the delay vector $\mathbf{w} \in\left\{\mathbf{w}_{1}, \ldots, \mathbf{w}_{N-d+1}\right\}$ of minimum velocity lying within a small distance $\delta$ of the diagonal. The upper panel of figure 3 shows the projection of the delay vectors for subject $\mathrm{B}$ onto the first two singular vectors of the global trajectory matrix, together with the estimate of $\overline{\mathbf{w}}$. The corresponding plot for subject A can be seen in the left panel of figure 9 . For both time series, the velocity of the reconstructed trajectory 
at $\mathbf{w}_{k}$ was approximated by $\left(\mathbf{w}_{k+1}-\mathbf{w}_{k}\right) / \tau$, and $\delta$ was set to be $\frac{1}{20}$ th of the radius of the reconstructed attractor, as estimated by the quantity $\max _{k}\left\|\mathbf{w}_{k}\right\|$. The delay vector obtained by carrying out the same calculation on the trajectory matrix for the model data can be seen in the left panel of figure 7 .

\subsection{Calculation of local dimension using SVD}

A common tool for analysing the reconstructed attractor in the neighbourhood of a given point $\mathbf{w} \in\left\{\mathbf{w}_{1}, \ldots, \mathbf{w}_{N-d+1}\right\}$ is the associated local trajectory matrix (Broomhead et al., 1987; Kirby, 2000). Given $\varepsilon>0$, let $B_{\varepsilon}(\mathbf{w})$ represent the set of points $\mathbf{w}_{k}$ for which $\left\|\mathbf{w}_{k}-\mathbf{w}\right\|<\varepsilon$; i.e. the open ball of radius $\varepsilon$ centred at $\mathbf{w}$. Then if there are $N_{\varepsilon}$ points in $B_{\varepsilon}(\mathbf{w})$, the local trajectory matrix $X_{\varepsilon}(\mathbf{w})$ is defined as the $N_{\varepsilon} \times d$ matrix whose rows consist of the vectors $\frac{1}{\sqrt{N_{\varepsilon}}}\left(\mathbf{w}_{k}-\mathbf{w}\right)^{T}$ with $\mathbf{w}_{k} \in B_{\varepsilon}(\mathbf{w})$. The SVD of $X_{\varepsilon}(\mathbf{w})$ provides useful information about the geometric structure of the reconstructed data in the neighbourhood of w (Broomhead et al., 1987; Broomhead and Jones, 1989; Kirby, 2000; Hundley and Kirby, 2003). Following the notation for the global trajectory matrix, the $j$ th singular value of $X_{\varepsilon}(\mathbf{w})$ will be written as $\sigma_{j}(\varepsilon)$ and the $j$ th singular vector as $\mathbf{c}_{j}(\varepsilon)$.

For sufficiently small $\varepsilon$, each vector of $B_{\varepsilon}(\mathbf{w})-\mathbf{w}$ lies in the tangent space $T_{\mathbf{w}} \mathbf{\Phi}(M)$ of the manifold $\boldsymbol{\Phi}(M)$ at $\mathbf{w}$. Also, for $1 \leq j \leq d, \sigma_{j}(\varepsilon)$ is the root mean square (RMS) projection of the vectors in $B_{\varepsilon}(\mathbf{w})-\mathbf{w}$ onto $\mathbf{c}_{j}(\varepsilon)$. Consequently, it is reasonable to assume that there are $m$ singular vectors of $X_{\varepsilon}(\mathbf{w})$ which span $T_{\mathbf{w}} \mathbf{\Phi}(M)$, for sufficiently small $\varepsilon$ (Broomhead et al., 1987; Hundley and Kirby, 2003). Write the indices of these vectors as $\left\{j_{1}, \ldots, j_{m}\right\}$, and the indices of the remaining $d-m$ vectors as $\left\{j_{m+1}, \ldots, j_{d}\right\}$. It then follows that in the limit $N_{\varepsilon} \rightarrow \infty$ of an isotropic, uniformly sampled neighbourhood of $\mathbf{w}$, $\sigma_{j_{k}}(\varepsilon) \sim \varepsilon+O\left(\varepsilon^{2}\right)$ for $1 \leq k \leq m$ and $\sigma_{j_{k}}(\varepsilon) \sim \varepsilon^{r(k)}+O\left(\varepsilon^{r(k)+1}\right)$ for $m+1 \leq k \leq d$, where $r(k) \geq 2$. In principle, the dimension $m$ of $M$ can therefore be determined by observing the scaling behaviour of the singular values as $\varepsilon$ is increased (Broomhead et al., 1987; Broomhead and Jones, 1989; Kirby, 2000; Hundley and Kirby, 2003).

In practice, however, noise and anisotropy of the data modify the scaling behaviour. In particular, additive white noise uncorrelated with the time series causes the singular values which scale nonlinearly in the noise-free case to scale linearly with $\varepsilon$, or to be independent of $\varepsilon$. What is actually seen depends on the size of $\varepsilon$ relative to the magnitude of the noise. When the data points spread beyond the extent of the ball in the direction of a particular singular vector, the corresponding singular value scales linearly with $\varepsilon$. Conversely, when $\varepsilon$ is greater than the extent of the data points in this direction, the corresponding singular value is independent of $\varepsilon$, with square approximately equal to the square of the noise-free value plus the variance of the noise. Similarly, even in the absence of noise, if the reconstructed attractor is thin in a particular direction - due, say, to a negative Lyapunov exponent of large modulus - when $\varepsilon$ is greater than the extent of the attractor in that direction, the corresponding singular value is independent of $\varepsilon$. An estimate of $m$ 
is therefore provided by the number of singular values $d_{L}$ that scale linearly with $\varepsilon$ or are constant above a level below which the singular values are believed to be dominated by noise, referred to as the noise floor (Broomhead et al., 1987; Broomhead and Jones, 1989; Healey et al., 1991). The quantity $d_{L}$ can in practice, however, both underestimate and overestimate $m$, depending on factors such as noise and strongly contracting Lyapunov exponents; it can be thought of more generally as the local dimension of the data at $\mathbf{w}$, since it is the minimum number of degrees of freedom needed to describe the data at that point.

\subsection{Estimating eigenvalues of the linearisation at a fixed point}

In the case where $\mathbf{w}$ lies close to a fixed point $\overline{\mathbf{w}}$ of the reconstructed dynamics, corresponding to a fixed point $\overline{\mathbf{z}}$ of $\dot{\mathbf{z}}=\mathbf{F}(\mathbf{z}), d_{L}$ eigenvalues of $D_{\mathbf{z}} \mathbf{F}(\overline{\mathbf{z}})$ can be estimated from a linear fit of the evolution under $\psi_{\tau}$ of the projections of the vectors in $B_{\varepsilon}(\mathbf{w})-\mathbf{w}$ onto the first $d_{L}$ singular vectors of $X_{\varepsilon}(\mathbf{w})$ (Healey et al., 1991). This can be seen easily in the case where the eigenvalues $\left\{\lambda_{1}, \ldots, \lambda_{D}\right\}$ of $D_{\mathbf{z}} \mathbf{F}(\overline{\mathbf{z}})$ are real and distinct. ${ }^{6}$ Let $\mathbf{u}_{i}$ be the eigenvector of $D_{\mathbf{z}} \mathbf{F}(\overline{\mathbf{z}})$ associated with $\lambda_{i}$, scaled to have unit norm, and write $\mathbf{z}_{k}-\overline{\mathbf{z}}=\sum_{i=1}^{D} Z_{i k} \mathbf{u}_{i}$. Then for sufficiently small $\varepsilon$, if $\mathbf{w}_{k}, \mathbf{w}_{k+1} \in B_{\varepsilon}(\mathbf{w})$, it follows from the linearisation of $\dot{\mathbf{z}}=\mathbf{F}(\mathbf{z})$ at $\overline{\mathbf{z}}$ and the Taylor expansion of $\boldsymbol{\Phi}$ about $\overline{\mathbf{z}}$ that

$$
\begin{aligned}
\mathbf{w}_{k}-\overline{\mathbf{w}} & \approx \sum_{i=1}^{D} Z_{i k} \tilde{\mathbf{u}}_{i} \\
\mathbf{w}_{k+1}-\overline{\mathbf{w}} & \approx \sum_{i=1}^{D} e^{\lambda_{i} \tau} Z_{i k} \tilde{\mathbf{u}}_{i}
\end{aligned}
$$

where $\tilde{\mathbf{u}}_{i}=D_{\mathbf{z}} \mathbf{\Phi}(\overline{\mathbf{z}}) \mathbf{u}_{i}$. Since, $\mathbf{w} \approx \overline{\mathbf{w}},(5)$ shows that each vector of $B_{\varepsilon}(\mathbf{w})-\mathbf{w}$ lies approximately in the linear space $L(\overline{\mathbf{w}})$ spanned by the columns of $D_{\mathbf{z}} \mathbf{\Phi}(\overline{\mathbf{z}})$. It can be shown that:

$$
\left\|\tilde{\mathbf{u}}_{i}\right\|_{1}=\left(\sum_{j=0}^{d-1} e^{j \lambda_{i} \tau}\right)\left|D_{\mathbf{z}} g(\overline{\mathbf{z}}) \mathbf{u}_{i}\right| .
$$

(7) and (5) therefore imply that if $\lambda_{i}<0$ with $\left|\lambda_{i}\right| \gg 0$ or $\left|D_{\mathbf{z}} g(\overline{\mathbf{z}}) \mathbf{u}_{i}\right| \approx 0, L(\overline{\mathbf{w}})$ will be thin in the direction of $\tilde{\mathbf{u}}_{i}$, in the sense that the relative variance of the data in the $\tilde{\mathbf{u}}_{i}$ direction will be small.

It is reasonable to assume that the thin directions will give rise to singular values of $X_{\varepsilon}(\mathbf{w})$ which have a magnitude commensurate with the noise floor. The other directions will give rise to singular values that scale linearly, or are constant, at values greater than the level of the noise. Relabelling the latter directions as $\left\{\tilde{\mathbf{u}}_{1}, \ldots, \tilde{\mathbf{u}}_{d_{L}}\right\}$, the points of $B_{\varepsilon}(\mathbf{w})-\mathbf{w}$ are thus effectively confined to the $d_{L}$-dimensional subspace $\hat{L}(\overline{\mathbf{w}})$ of $L(\overline{\mathbf{w}})$ spanned by $\left\{\tilde{\mathbf{u}}_{1}, \ldots, \tilde{\mathbf{u}}_{d_{L}}\right\}$, with the first $d_{L}$ singular vectors $\left\{\mathbf{c}_{1}(\varepsilon), \ldots, \mathbf{c}_{d_{L}}(\varepsilon)\right\}$ of $X_{\varepsilon}(\mathbf{w})$

\footnotetext{
${ }^{6}$ It is straightforward to extend this analysis to the case where $D_{\mathbf{z}} \mathbf{F}(\overline{\mathbf{z}})$ has complex eigenvalues.
} 
approximating a basis for $\hat{L}(\overline{\mathbf{w}})$. When $\mathbf{w}$ lies close to a fixed point of the dynamics, the local dimension thus computes the number of significant eigendirections of the linearisation, which may differ from $m$.

As the points of $B_{\varepsilon}(\mathbf{w})-\mathbf{w}$ effectively lie in a proper subset of the delay space $\mathbb{R}^{d}$, attempting to estimate eigenvalues of $D_{\mathbf{z}} \mathbf{F}(\overline{\mathbf{z}})$ directly from pairs $\mathbf{w}_{k}-\mathbf{w}, \mathbf{w}_{k+1}-\mathbf{w} \in$ $B_{\varepsilon}(\mathbf{w})-\mathbf{w}$ is an ill-posed numerical problem. The eigenvalues $\left\{\lambda_{1}, \ldots, \lambda_{d_{L}}\right\}$ associated with $\left\{\mathbf{u}_{1}, \ldots, \mathbf{u}_{d_{L}}\right\}$ can, however, be robustly estimated by projecting the points of $B_{\varepsilon}(\mathbf{w})-\mathbf{w}$ onto $\hat{L}(\overline{\mathbf{w}})$ (Healey et al., 1991). Define $\hat{\mathbf{w}}_{k}$ to be the projection of $\mathbf{w}_{k}-\mathbf{w}$ onto the basis $\left\{\mathbf{c}_{1}(\varepsilon), \ldots, \mathbf{c}_{d_{L}}(\varepsilon)\right\}$, so that $\hat{\mathbf{w}}_{k}=C_{\varepsilon}^{T}\left(\mathbf{w}_{k}-\mathbf{w}\right)$, where $C_{\varepsilon}$ is the $d \times d_{L}$ matrix whose $j$ th column is $\mathbf{c}_{j}(\varepsilon)$. It then follows from (5) and (6) (together with the approximation $\mathbf{w} \approx \overline{\mathbf{w}})$, that $\hat{\mathbf{w}}_{k+1} \approx \Lambda \hat{\mathbf{w}}_{k}$, where

$$
\Lambda=\left(C_{\varepsilon}^{T} \tilde{U}\right) \operatorname{diag}\left\{e^{\lambda_{1} \tau}, \ldots, e^{\lambda_{d_{L}} \tau}\right\}\left(C_{\varepsilon}^{T} \tilde{U}\right)^{-1}
$$

and $\tilde{U}$ is the $d \times d_{L}$ matrix $\left[\tilde{\mathbf{u}}_{1}, \ldots, \tilde{\mathbf{u}}_{d_{L}}\right]$. Writing $\left\{\mu_{1}, \ldots, \mu_{d_{L}}\right\}$ for the eigenvalues of $\Lambda$, the form of $\Lambda$ implies that $\lambda_{j}=\frac{1}{\tau} \ln \left(\mu_{j}\right)$ for $1 \leq j \leq d_{L}$. Estimates of $\left\{\lambda_{1}, \ldots, \lambda_{d_{L}}\right\}$ can therefore be obtained from an estimate of $\Lambda$.

In practice, $\Lambda$ is estimated by collecting a subset

$$
\left\{\left(\hat{\mathbf{w}}_{f_{1}}, \hat{\mathbf{w}}_{f_{1}+1}\right), \ldots,\left(\hat{\mathbf{w}}_{f_{F_{N}}}, \hat{\mathbf{w}}_{f_{F_{N}}+1}\right)\right\}
$$

of the $S_{N}$ pairs of projected delay vectors $\left\{\hat{\mathbf{w}}_{k}, \hat{\mathbf{w}}_{k+1}\right\}$ for which $\mathbf{w}_{k}, \mathbf{w}_{k+1} \in B_{\varepsilon}(\mathbf{w})$. The approximation $\hat{\mathbf{w}}_{f_{j}+1} \approx \Lambda \hat{\mathbf{w}}_{f_{j}}$ implies that $W_{2} \approx W_{1} \Lambda^{T}$, where $W_{1}$ and $W_{2}$ are the $F_{N} \times$ $d_{L}$ matrices with $j$ th rows $\hat{\mathbf{w}}_{f_{j}}^{T}$ and $\hat{\mathbf{w}}_{f_{j}+1}^{T}$ respectively. $\Lambda$ can therefore be estimated by finding the matrix $Y$ which solves the least-squares problem $\min _{Y \in \mathbb{R}^{d_{L} \times d_{L}}}\left\|W_{2}-W_{1} Y\right\|_{2}^{2}$. By construction $W_{1}$ is full rank, giving the following least-squares estimate $\Lambda_{L S}$ of $\Lambda$ (Barnett, 1990):

$$
\Lambda_{L S}=\left(W_{2}^{T} W_{1}\right)\left(W_{1}^{T} W_{1}\right)^{-1}
$$

The remaining $T_{N}=S_{N}-F_{N}$ pairs of projected delay vectors $\left\{\hat{\mathbf{w}}_{k}, \hat{\mathbf{w}}_{k+1}\right\}$ with $\mathbf{w}_{k}, \mathbf{w}_{k+1} \in$ $B_{\varepsilon}(\mathbf{w})$ are used to test the fit. These will be written as

$$
\left\{\left(\hat{\mathbf{w}}_{c_{1}}, \hat{\mathbf{w}}_{c_{1}+1}\right), \ldots,\left(\hat{\mathbf{w}}_{c_{T_{N}}}, \hat{\mathbf{w}}_{c_{T_{N}}+1}\right)\right\}
$$

In summary, it is possible to estimate a given eigenvalue $\lambda_{i}$ of $D_{\mathbf{z}} \mathbf{F}(\overline{\mathbf{z}})$ from the delay vectors $\mathbf{w}_{k}$ lying in the neighbourhood of $\overline{\mathbf{w}}$, unless $\lambda_{i}$ is large and negative or $D_{\mathbf{z}} g(\overline{\mathbf{z}})$ is close to being orthogonal to the eigenvector of $D_{\mathbf{z}} \mathbf{F}(\overline{\mathbf{z}})$ corresponding to $\lambda_{i}$. The eigenvalues of $D_{\mathbf{z}} \mathbf{F}(\overline{\mathbf{z}})$ which can be estimated from the data will be referred to here as the observable eigenvalues, and will be assumed to be those that effectively determine the local behaviour of $\dot{\mathbf{z}}=\mathbf{F}(\mathbf{z})$ in the neighbourhood of $\overline{\mathbf{z}}$. 


\subsection{Calculation of local dimension and eigenvalues of linearisation at the fixed point estimates}

\subsubsection{Results for the experimental data}

The scaling of the local singular values with the ball radius $\varepsilon$ at the estimate of the hypothetical fixed point $\overline{\mathbf{w}}$ is shown in figure 4 for both experimental time series. In order to compute the local dimension $d_{L}$ from the singular values, it is necessary to obtain an estimate of the noise floor. Under the assumption that the observational noise introduced by the IRIS 6500 is additive and white, the ratio of the resolution $\left(0.03^{\circ}\right)$ to the standard deviation of the unnormalised data was taken as the noise floor estimate, written $\sigma_{n}$. This gave the values $\sigma_{n}=0.0641$ for subject $\mathrm{A}$ and $\sigma_{n}=0.0232$ for subject $\mathrm{B}$. For both $\mathrm{A}$ and B there are three singular values lying above $\sigma_{n}$ which scale linearly with $\varepsilon$ or are constant, giving a local dimension $d_{L}$ of 3 (see figure 4 ).

Following the computation of $d_{L}$, estimates $\left\{\hat{\lambda}_{1}, \hat{\lambda}_{2}, \hat{\lambda}_{3}\right\}$ of the observable eigenvalues $\left\{\lambda_{1}, \lambda_{2}, \lambda_{3}\right\}$ of $D_{\mathbf{z}} \mathbf{F}(\overline{\mathbf{z}})$ were obtained from points lying within an $\varepsilon$-ball of the fixed point estimate over a range of $\varepsilon$ values. For each choice of $\varepsilon, 75 \%$ of the pairs $\left\{\hat{\mathbf{w}}_{k}, \hat{\mathbf{w}}_{k+1}\right\}$ with $\mathbf{w}_{k}, \mathbf{w}_{k+1} \in B_{\varepsilon}(\mathbf{w})$ were used to calculate the least-squares fit $\Lambda_{L S}$ to the map $\Lambda$, while the remaining $25 \%$ were used to test the accuracy of the fit. Over the chosen $\varepsilon$ ranges, the normalised RMS error

$$
E_{F}=\frac{1}{\varepsilon} \sqrt{\frac{1}{F_{N}} \sum_{j=1}^{F_{N}}\left\|\hat{\mathbf{w}}_{f_{j}+1}-\Lambda_{L S} \hat{\mathbf{w}}_{f_{j}}\right\|_{2}^{2}}
$$

for the fit vectors was found to be comparable to the normalised RMS error

$$
E_{T}=\frac{1}{\varepsilon} \sqrt{\frac{1}{T_{N}} \sum_{j=1}^{T_{N}}\left\|\hat{\mathbf{w}}_{c_{j}+1}-\Lambda_{L S} \hat{\mathbf{w}}_{c_{j}}\right\|_{2}^{2}}
$$

for the test vectors, with maximum values of $E_{F}=0.0683, E_{T}=0.0755$ for subject A, and $E_{F}=0.0477, E_{T}=0.0400$ for subject B. The estimates were real-valued over all the $\varepsilon$ ranges considered. After each calculation of $\Lambda_{L S}$, the $\hat{\lambda}_{i}$ s were sorted so that $\hat{\lambda}_{1}>\hat{\lambda}_{2}>\hat{\lambda}_{3}$.

The accuracy of the estimates was gauged by employing a simple bootstrap technique to approximate the probability distributions of the $\hat{\lambda}_{i}$ s about the true values. For each choice of $\varepsilon, 5000$ Monte Carlo samples $\left\{\hat{\lambda}_{1}^{(p)}, \hat{\lambda}_{2}^{(p)}, \hat{\lambda}_{3}^{(p)}\right\}$ were generated. Each Monte Carlo sample was obtained by calculating $\Lambda_{L S}$ after using a uniform random number generator to remove a random fraction of the $F_{N}$ pairs used for the least-squares fit. For each $1 \leq i \leq 3$, the distribution of $\hat{\lambda}_{i}^{(p)}$ about $\hat{\lambda}_{i}$ was taken to reflect the underlying distribution of $\hat{\lambda}_{i}$ about $\lambda_{i}$, enabling confidence intervals to be constructed (Press et al., 1992). Figure 5 shows the $\hat{\lambda}_{i}$ values obtained over the $\varepsilon$ ranges considered. Also shown are the $95 \%$ confidence intervals

$$
\left(\hat{\lambda}_{i}-1.96 \sigma\left(\hat{\lambda}_{i}^{p}-\hat{\lambda}_{i}\right), \hat{\lambda}_{i}+1.96 \sigma\left(\hat{\lambda}_{i}^{p}-\hat{\lambda}_{i}\right)\right)
$$


calculated from the bootstrap samples. (Here, and thereafter, $\sigma($.$) denotes the standard$ deviation of its argument).

It can be seen that for both subjects, the eigenvalue estimates and confidence intervals are stable over the chosen $\varepsilon$ ranges. The final estimates of $\left\{\lambda_{1}, \lambda_{2}, \lambda_{3}\right\}$ were taken to be those computed for the value of $\varepsilon$ giving the smallest fit error. These are given in table 1 , together with the $95 \%$ confidence interval and normalised standard error $\hat{\lambda}_{i}^{E}=\frac{\sigma\left(\hat{\lambda}_{2}^{(p)}-\hat{\lambda}_{2}\right)}{\left|\hat{\lambda}_{2}\right|}$ for each estimate, and the corresponding fit and prediction errors.

For both experimental data sets, the small RMS errors for the fit and test vectors coupled with the stability of the eigenvalue estimates over a range of ball radii are good evidence for a fixed point $\overline{\mathbf{w}}$ of the reconstructed dynamics. In addition, the points in the time series corresponding to $\overline{\mathbf{w}}$ coincide with foveation (cf. the right panel of figure $9)$. The evidence for $\overline{\mathbf{w}}$ thus suggests the existence of a fixed point $\overline{\mathbf{z}}=\boldsymbol{\Phi}^{-1}(\overline{\mathbf{w}})$ of the unforced oculomotor dynamics $\dot{\mathbf{z}}=\mathbf{F}$ (z) representing stationary gaze at the primary position, hypothesised in section 4 . The confidence intervals for the eigenvalue estimates strongly suggest that $\lambda_{1}>0$ and $\lambda_{3}<0$, with $\left|\lambda_{3}\right| \gg\left|\lambda_{1}\right| \gg 0$. Plots showing the eigenvectors $\left\{\mathbf{e}_{1}, \mathbf{e}_{2}, \mathbf{e}_{3}\right\}$ of the least-squares fit $\Lambda_{L S}$ to $\Lambda$ together with the points $\hat{\mathbf{w}}_{k}$ used to compute $\Lambda_{L S}$ enable the negative eigenvalue $\lambda_{3}$ to be identified with the fast phase of the jerk oscillation, that is the saccadic movement which returns the retinal image to the vicinity of the fovea. The positive eigenvalue $\lambda_{1}$ appears to correspond to the slow phase, the low velocity movement of the retinal image away from the foveal region. The identification of $\lambda_{1}$ and $\lambda_{3}$ with the slow and fast phases is illustrated in the lower panel of figure 3 , which shows plots of $\hat{\mathbf{w}}_{k}$ and $\left\{\mathbf{e}_{1}, \mathbf{e}_{2}, \mathbf{e}_{3}\right\}$ for the choice of ball radius used to obtain the final eigenvalue estimates from the time series of subject $\mathrm{B}$.

In contrast to the positive assertions that can be made regarding the signs of $\lambda_{1}$ and $\lambda_{3}$, for both subjects the sign of $\lambda_{2}$ cannot be unambiguously determined since the confidence intervals for $\hat{\lambda}_{2}$ contain 0 . The estimate $\hat{\mu}_{2}$ of the eigenvalue $\mu_{2}$ of $\Lambda$ is equal to $0.9998 \pm$ 0.0340 for subject $\mathrm{A}$ and $0.9935 \pm 0.0104$ for subject B (errors represent the $95 \%$ confidence intervals). These values suggest that $\mu_{2} \approx 1$ in both cases, corresponding to a direction of the dynamics in the neighbourhood of $\overline{\mathbf{z}}$ along which trajectories have velocity close to zero (cf. the orientation of $\mathbf{e}_{2}$ in the lower panel of figure 3 ). Since $\hat{\lambda}_{2}=\frac{1}{\tau} \ln \left(\hat{\mu}_{2}\right)$, it seems reasonable to infer that $\lambda_{2} \approx 0$, with a consequently high normalised standard error $\hat{\lambda}_{2}^{E}$ (see table 1). Indeed, it is clear that even moderate noise will cause perturbations to the estimates of $\mu_{2}$ that will preclude the possibility of estimating $\lambda_{2}$ to a sufficiently high accuracy to determine whether $\lambda_{2}>0$ or $\lambda_{2}<0$.

\subsubsection{Results for the model data}

The singular value scaling at the delay vector obtained by using the fixed point estimation method on the unstable NI model data can be seen in the top left panel of figure 8 . As for the experimental data, the ratio of the IRIS 6500 resolution to the standard deviation of 
the unnormalised time series was taken as an estimate of the noise floor $\sigma_{n}$, yielding the value $\sigma_{n}=0.0186$. There are two singular values lying above $\sigma_{n}$ which scale linearly with $\varepsilon$, giving a local dimension $d_{L}$ of 2 . This contrasts with the value 3 of $d_{L}$ observed at the fixed point estimates for the experimental time series.

Table 2 gives the final estimates $\left\{\hat{\eta}_{1}, \hat{\eta}_{2}\right\}$ of the eigenvalues $\left\{\eta_{1}, \eta_{2}\right\}$ of the matrix $\ln \left(\Lambda^{\frac{1}{\tau}}\right)$; these quantities are the result of applying the same procedure that was used to estimate the observable eigenvalues of $D_{\mathbf{z}} \mathbf{F}(\overline{\mathbf{z}})$ from the experimental time series. The 95\% confidence intervals calculated from 5000 Monte Carlo samples $\left\{\hat{\eta}_{1}^{(p)}, \hat{\eta}_{2}^{(p)}\right\}$ generated using the bootstrap technique described in the previous section are also shown in the table, together with the normalised standard error $\hat{\eta}_{i}^{E}=\frac{\sigma\left(\hat{\eta}_{2}^{(p)}-\hat{\eta}_{2}\right)}{\left|\hat{\eta}_{2}\right|}$ of each estimate, and the fit and prediction errors. The small values of $E_{F}$ and $E_{T}$ indicate that, as for the experimental data, the dynamics during the low velocity phase of the cycle can be well approximated with a linear model. Moreover, the confidence intervals clearly imply that $\eta_{1}>0$ and $\eta_{2}<0$. Plots of the eigenvectors of $\Lambda$ show that $\eta_{1}$ corresponds to the drift resulting from the unstable NI while $\eta_{2}$ corresponds to the corrective saccades which refixate the target. The unstable NI model can thus account for two of the eigenvalues observed in the experimental data, $\lambda_{1}$ and $\lambda_{3}$, but not the near-zero eigenvalue $\lambda_{2}$.

\subsubsection{Interpretation of the eigenvalue estimates}

The eigenvalue estimates for the jerk CN data suggests that $D_{\mathbf{z}} \mathbf{F}(\overline{\mathbf{z}})$ possesses at least one positive eigenvalue. This result is consistent with $\overline{\mathbf{z}}$ having lost stability through a bifurcation, as proposed in section 4 . As stated therein, the eigenvalues of $D_{\mathbf{z}} \mathbf{F}(\overline{\mathbf{z}})$ are the eigenvalues of the muscle plant together with the NI eigenvalue $-\frac{1}{N_{T}}$, which is of the order of -0.04 in normal subjects, and the eigenvalues of the OCS derivative $D_{\mathbf{y}} \mathbf{f}(\overline{\mathbf{y}})$. Moreover, since the muscle plant eigenvalues are negative, $\overline{\mathbf{z}}$ can only lose stability as a consequence of $N_{T}$ becoming negative, or $D_{\mathbf{y}} \mathbf{f}(\overline{\mathbf{y}})$ developing an eigenvalue with positive real part. The identification of $\lambda_{1}$ with the destabilising slow phase together with the fact the eigenvalue spectrum suggests a codimension 1 bifurcation means the most likely scenario is that $\overline{\mathbf{z}}$ has lost stability through a 1-dimensional bifurcation in the OCS, yielding the positive eigenvalue $\lambda_{1}$, and $\lambda_{2}$ is negative $\left(N_{T}>0\right)$, reflecting a normal, stable integrator. The inability of the unstable NI to account for the near-zero eigenvalue provides further evidence for this hypothesis. The eigenvalue estimates thus suggest that the origin of the bifurcation is one of the oculomotor subsystems rather than the NI.

\subsection{Construction of a Poincaré map incorporating a fast phase modelled as a homoclinic reinjection}

Control models of jerk $\mathrm{CN}$ assume that the fast phase of the oscillation is a saccadic correction of the ocular drift away from the target. An error-correcting saccade is activated 
when the eye position or velocity crosses a threshold level (Optican and Zee, 1984; Tusa et al., 1992; Dell'Osso, 2002; Jacobs and Dell'Osso, 2004). In models based on nonlinear dynamics, the fast phase is a consequence of the orientation of a slow manifold in the system phase space. The sections of trajectories representing the fast phase are constrained to the slow manifold, which intersects a fixed point representing fixation of the target. As a consequence, the fast phases are forced to terminate close to the target position (Broomhead et al., 2000; Akman et al., 2005). Both types of models thus result in a fast phase which is a deterministic refixation of the target. Within the framework of the generalised oculomotor model $\dot{\mathbf{z}}=\mathbf{F}(\mathbf{z})$, the simplest object associated with such a mechanism is an orbit $\tilde{\Gamma}$ that is nearly homoclinic to $\overline{\mathbf{z}}$. i.e. an orbit which lies close to a trajectory $\Gamma$ such that $\phi_{t}(\mathbf{z}) \rightarrow \overline{\mathbf{z}}$ as $t \rightarrow \infty$ and as $t \rightarrow-\infty$ for $\mathbf{z} \in \Gamma$. The existence of $\tilde{\Gamma}$ ensures that points lying sufficiently close to $\overline{\mathbf{z}}$ at the beginning of a CN cycle will be reinjected back into the neighbourhood of $\overline{\mathbf{z}}$ at the end of the cycle. As $\overline{\mathbf{z}}$ represents the eye lying at rest in the primary position, the reinjection induced by $\tilde{\Gamma}$ models a refixating fast phase.

Combining this assumption of a near-homoclinic orbit with the eigenvalue estimates enables the construction of a Poincaré map; that is a function $\mathbf{P}$ defined on a set $\Sigma$ intersecting the flow of $\dot{\mathbf{z}}=\mathbf{F}(\mathbf{z})$ transversally which maps the point at which a trajectory first intersects $\Sigma$ to the point at which it next intersects $\Sigma$. The analysis of the map $\mathbf{P}$ provides information on the behaviour of the full higher-dimensional flow. In particular, stable and unstable fixed points of $\mathbf{P}$ give rise respectively to stable and unstable periodic orbits of $\dot{\mathbf{z}}=\mathbf{F}(\mathbf{z})$ that pass through $\Sigma$ (Guckenheimer and Holmes, 1983; Glendinning, 1994; Alligood et al., 1996).

Following (Guckenheimer and Holmes, 1983) and (Glendinning, 1994), an explicit expression for such a map $\mathbf{P}$ can be obtained by expressing $\mathbf{P}$ as the composition of two maps; a map $\mathbf{P}_{1}$ obtained from the dynamics in the neighbourhood of $\overline{\mathbf{z}}$ - corresponding to the motion of the eye close to foveation - and a map constructed by considering the dynamics outside this neighbourhood - corresponding to the reinjection mechanism which brings the image of the target back into the vicinity of the fovea at the end of the $\mathrm{CN}$ cycle. The relative magnitudes of the eigenvalues then imply that $\dot{\mathbf{z}}=\mathbf{F}(\mathbf{z})$ should possess a limit cycle attractor, as will now be shown.

\section{Derivation of $\mathbf{P}$}

Poincaré's Linearisation Theorem states that it is possible to choose a coordinate transformation such that the dynamics in the neighbourhood of $\overline{\mathbf{z}}$ are the same as the linearised dynamics (Glendinning, 1994). Assume that this transformation has been applied after shifting $\overline{\mathbf{z}}$ to the origin $\mathbf{0}$. The flow in the neighbourhood of $\mathbf{0}$ can then be approximated by the flow in the 3-dimensional space spanned by the eigenvectors of the transformed vector field associated with the observable eigenvalues $\left\{\lambda_{1}, \lambda_{2}, \lambda_{3}\right\}$ of $D_{\mathbf{z}} \mathbf{F}(\overline{\mathbf{z}})$. Writing 
$\left(X_{1}, X_{2}, X_{3}\right)$ for the coordinates in the eigenvector basis, the dynamics are thus given by:

$$
X_{i}(t)=e^{\lambda_{i} t} X_{i}(0): 1 \leq i \leq 3 .
$$

By rescaling the $X_{i}$ s, it can be assumed that the neighbourhood within which this approximation holds lies in the cube $\left\{\left|X_{i}\right| \leq 1: 1 \leq i \leq 3\right\}$ (see figure 6). Let $\Sigma$ be the side of the cube defined by $X_{3}=1$ and $\Sigma^{\prime}$ the side defined by $X_{1}=1$. Since $\lambda_{1}>0>\lambda_{2} \gg \lambda_{3}$, trajectories intersect $\Sigma$ as they enter the neighbourhood of $\mathbf{0}$ (see the lower panel of figure 3 ). If the $X_{1}$ coordinate of the point at which a given trajectory intersects $\Sigma$ is positive, the trajectory intersects $\Sigma^{\prime}$ as it exits the neighbourhood of $\mathbf{0}$, while if it is negative, it intersects the side of the cube opposite $\Sigma^{\prime}$ (see figure 6).

Define $\mathbf{P}_{1}$ to be the map which takes points on $\Sigma$ to points on $\Sigma^{\prime}$. (8) implies that the time $\bar{t}$ taken for a point with $X_{1}>0$ to be mapped from $\Sigma$ to $\Sigma^{\prime}$ by $\mathbf{P}_{1}$ is

$$
\bar{t}=\frac{1}{\lambda_{1}} \ln \left(\frac{1}{X_{1}}\right) .
$$

Writing the coordinates on $\Sigma^{\prime}$ as $\left(X_{3}, X_{2}\right)$, it then follows from (8) that $\mathbf{P}_{1}$ has the form

$$
\mathbf{P}_{1}\left(X_{1}, X_{2}\right)=\left(\begin{array}{c}
X_{1}^{\delta_{1}} \\
X_{1}^{\delta_{2}} X_{2}
\end{array}\right)
$$

where $\delta_{1}=-\frac{\lambda_{3}}{\lambda_{1}}$ and $\delta_{2}=-\frac{\lambda_{2}}{\lambda_{1}}$. Next, define $\mathbf{P}_{2}$ to be the map which takes points on $\Sigma^{\prime}$ to points on $\Sigma$. i.e. the map induced by the near-homoclinic orbit $\tilde{\Gamma}$. Since the $X_{1}$-axis is the unstable manifold of the origin, it can be assumed that $\tilde{\Gamma}$ intersects $\Sigma^{\prime}$ at $(0,0)$. Approximating $\mathbf{P}_{2}$ by its Taylor expansion about $(0,0)$ yields

$$
\mathbf{P}_{2}\left(X_{3}, X_{2}\right)=E\left(\begin{array}{c}
X_{3} \\
X_{2}
\end{array}\right)+\mathbf{e},
$$

where the $2 \times 2$ matrix $E$ represents the Jacobian derivative of $\mathbf{P}_{2}$ evaluated at $(0,0)$, and $\mathbf{e}=\mathbf{P}_{2}(0,0)$ (Guckenheimer and Holmes, 1983; Glendinning, 1994). $\mathbf{P}_{2}$ is an affine mapping which sends a disc of points centred at $(0,0)$ on $\Sigma^{\prime}$ to a distorted disc of points centred at $\mathbf{e}$ on $\Sigma$ (see figure 6 ). e can thus be thought of as the error in the correction to the drift of the eye away from the target produced by the fast phase. Composing $\mathbf{P}_{2}$ with $\mathbf{P}_{1}$ gives the required Poincaré map $\mathbf{P}$. Substituting (10) and (11) into $\mathbf{P}=\mathbf{P}_{2} \circ \mathbf{P}_{1}$ leads to the expression below:

$$
\mathbf{P}\left(X_{1}, X_{2}\right)=E\left(\begin{array}{c}
X_{1}^{\delta_{1}} \\
X_{1}^{\delta_{2}} X_{2}
\end{array}\right)+\mathbf{e}
$$

\section{Simplification of $\mathbf{P}$}

Using the fact that the time-of-flight $\bar{t}$ is bounded enables $\mathbf{P}$ to be simplified. $\bar{t}$ must be bounded above by some value $t_{2}$, reflecting the minimum frequency of the nystagmus, and 
bounded below by some value $t_{1}$, reflecting the minimum residency time of the target image in the foveal region. Writing $\alpha=e^{-\lambda_{1} t_{2}}$ and $\beta=e^{-\lambda_{1} t_{1}}$, (9) implies that on the domain of $\mathbf{P}, \alpha \leq X_{1} \leq \beta$ and hence $\alpha^{\delta_{2}} \leq X_{1}^{\delta_{2}} \leq \beta^{\delta_{2}}$. Computing $t_{1}$ and $t_{2}$ from the time series gives the values $\alpha=0.0040, \beta=0.4405$ for subject $\mathrm{A}$ and $\alpha=0.0392, \beta=0.4925$ for subject B (Akman, 2000). Setting $\lambda_{2}$ equal to $-\frac{1}{N_{T}}$ with the standard NI time constant of $25 \mathrm{~s}$ yields $\delta_{2}=9.7580 \times 10^{-4}$ for $\mathrm{A}$, and $\delta_{2}=19.7667 \times 10^{-4}$ for B. For both subjects, these values imply $\alpha^{\delta_{2}}, \beta^{\delta_{2}} \approx 1$. This gives $X_{1}^{\delta_{2}} \approx 1$, whence the final approximation

$$
\mathbf{P}\left(X_{1}, X_{2}\right)=E\left(\begin{array}{c}
X_{1}^{\delta_{1}} \\
X_{2}
\end{array}\right)+\mathbf{e} .
$$

Defining $\mathbf{X}=\left(X_{1}, X_{2}\right)^{T}$ and $\mathbf{p}(\mathbf{X})=\left(X_{1}^{\delta_{1}}, X_{2}\right)^{T}$ enables (12) to be written in the vectorised form

$$
\mathbf{P}(\mathbf{X})=E \mathbf{p}(\mathbf{X})+\mathbf{e}
$$

\section{Analysis of $\mathbf{P}$}

As $\mathbf{P}$ is a return map, it must map points in its domain back onto $\Sigma$. This places restrictions on $E$ which can be used to deduce that $\mathbf{P}$ is a contraction mapping on a closed subset of its domain. The condition on $\mathbf{P}$ can be expressed as $\|\mathbf{P}(\mathbf{X})\|_{\infty} \leq 1$ for all $\mathbf{X}$ in the domain of $\mathbf{P}$, where $\|.\|_{\infty}$ represents the vector and matrix infinity norm. (13) implies

$$
\|\mathbf{P}(\mathbf{X})\|_{\infty} \leq\|E\|_{\infty}\|\mathbf{p}(\mathbf{X})\|_{\infty}+\|\mathbf{e}\|_{\infty} \leq\|E\|_{\infty} \max _{\mathbf{X} \in \Sigma, \alpha \leq X_{1} \leq \beta}\|\mathbf{p}(\mathbf{X})\|_{\infty}+\|\mathbf{e}\|_{\infty}
$$

By definition, $\|\mathbf{p}(\mathbf{X})\|_{\infty}=\max \left\{X_{1}^{\delta_{1}},\left|X_{2}\right|\right\}$. Since $-\lambda_{3} \gg \lambda_{1}, \delta_{1}>1$ and thus for $\mathbf{X} \in \Sigma$ with $\alpha \leq X_{1} \leq \beta,\left|X_{1}^{\delta_{1}}\right| \leq \beta^{\delta_{1}}<1$. As $\left|X_{2}\right| \leq 1$ on $\Sigma, \max _{\mathbf{X} \in \Sigma, \alpha \leq X_{1} \leq \beta}\|\mathbf{p}(\mathbf{X})\|_{\infty}=1$. A minimally sufficient condition for $\mathbf{P}$ to map its domain into $\Sigma$ is therefore $\|E\|_{\infty} \leq 1-\|\mathbf{e}\|_{\infty}$.

So now let $\mathbf{X}=\left(X_{1}, X_{2}\right)^{T}$ and $\mathbf{X}^{\prime}=\left(X_{1}^{\prime}, X_{2}^{\prime}\right)^{T}$ lie in the domain of $\mathbf{P}$. Then by (13),

$$
\mathbf{P}(\mathbf{X})-\mathbf{P}\left(\mathbf{X}^{\prime}\right)=E\left(\mathbf{p}(\mathbf{X})-\mathbf{p}\left(\mathbf{X}^{\prime}\right)\right)
$$

Using the Mean Value Theorem, the expression above can be written as

$$
\mathbf{P}(\mathbf{X})-\mathbf{P}\left(\mathbf{X}^{\prime}\right)=E J(\xi)\left(\mathbf{X}-\mathbf{X}^{\prime}\right)
$$

where $J(\xi)$ is the Jacobian derivative of $\mathbf{p}$ evaluated at a point $\xi=\left(\xi_{1}, \xi_{2}\right)^{T}$ lying on the line segment between $\mathbf{X}$ and $\mathbf{X}^{\prime} . J=\operatorname{diag}\left\{\delta_{1} \xi_{1}^{\delta_{1}-1}, 1\right\}$, and so the expression above implies

$$
\begin{aligned}
\left\|\mathbf{P}(\mathbf{X})-\mathbf{P}\left(\mathbf{X}^{\prime}\right)\right\|_{\infty} & \leq\|E\|_{\infty} \max \left\{\delta_{1} \xi_{1}^{\delta_{1}-1}, 1\right\}\left\|\mathbf{X}-\mathbf{X}^{\prime}\right\|_{\infty} \\
& \leq\left(1-\|\mathbf{e}\|_{\infty}\right) \max \left\{\delta_{1} \beta^{\delta_{1}-1}, 1\right\}\left\|\mathbf{X}-\mathbf{X}^{\prime}\right\|_{\infty}
\end{aligned}
$$


The eigenvalue ratio $\delta_{1}$ has the value 7.0886 for subject $\mathrm{A}$ and 11.2025 for subject $\mathrm{B}$, giving $\delta_{1} \beta^{\delta_{1}-1}=0.0482$ for $\mathrm{A}$ and $\delta_{1} \beta^{\delta_{1}-1}=0.0081$ for B. Hence, $\max \left\{\delta_{1} \beta^{\delta_{1}-1}, 1\right\}=1$ in both cases, leading to the final inequality

$$
\left\|\mathbf{P}(\mathbf{X})-\mathbf{P}\left(\mathbf{X}^{\prime}\right)\right\|_{\infty} \leq\left(1-\|\mathbf{e}\|_{\infty}\right)\left\|\mathbf{X}-\mathbf{X}^{\prime}\right\|_{\infty}
$$

Finally, let $\bar{\Sigma}$ be the largest closed subset of the domain of $\mathbf{P}$ such that $\mathbf{P}(\bar{\Sigma}) \subseteq \bar{\Sigma}$. Since $0<1-\|\mathbf{e}\|_{\infty}<1, \mathbf{P}$ is a contraction mapping on $\bar{\Sigma}$, and so has a unique, globally attracting fixed point in this set. It follows that there is an attracting periodic orbit of $\dot{\mathbf{z}}=\mathbf{F}(\mathbf{z})$ in a tubular neighbourhood of $\tilde{\Gamma}$. The attractor $\mathcal{A}$ of $\dot{\mathbf{z}}=\mathbf{F}(\mathbf{z})$ should therefore be a limit cycle, giving a periodic eye position time series.

\subsection{Calculation of local dimension around the jerk CN cycle}

The simplest attractor $\mathcal{A}$ of $\dot{\mathbf{z}}=\mathbf{F}(\mathbf{z})$ that could produce oscillatory behaviour is a stable limit cycle; indeed it was argued in the last section that a fast phase comprising a homoclinic refixation will lead to an attractor of this type. For such an attractor, the finite dimensional manifold $M$ containing $\mathcal{A}$ is $\mathcal{A}$ itself, and so the dimension of $M$ is 1 . It therefore follows from the discussion of sections 6.2 and 6.3 that for both experimental data sets, $d_{L}$ should equal 1 on the reconstructed trajectory, except at points in the vicinity of the reconstructed fixed point $\overline{\mathbf{w}}$, where $d_{L}$ reflects the dimension of the linearised dynamics rather than of $M$. Since, the local dimension is 3 at $\overline{\mathbf{w}}$ (see figure 4 ), $d_{L}$ should therefore decrease towards 1 as the trajectory leaves the neighbourhood of $\overline{\mathbf{w}}$ during the slow phase, before increasing back to 3 towards the end of the fast phase as the trajectory reenters the vicinity of the fixed point. Indeed, a variation in $d_{L}$ of this type was observed for the unstable NI model data, for which the underlying attractor is periodic (Optican and Zee, 1984). Figure 7 shows 3 points of the reconstructed attractor bounded away from the foveation region at which $d_{L}$ was computed. The scaling of the local singular values at each point is presented in figure 8 . The singular value plots show that $d_{L}$ decreases from 2 at foveation to 1 at the beginning of the fast phase, before increasing back to 2 at the end of the fast phase.

In order to determine whether dimension changes consistent with a limit cycle occur in the experimental data sets, $d_{L}$ was calculated at 3 points of the reconstructed attractors roughly coincident in terms of cycle phase with those used for the model data. The points chosen for the time series of subject A are shown in figure 9, while the corresponding local singular value plots are presented in figure 10 . It can be seen that $d_{L}$ decreases to 2 early in the slow phase, increases to 4 as the cycle enters the fast phase, before decreasing back to 3 as the fast phase terminates. The same variation in $d_{L}$ was observed for the time series of subject B. The increase in $d_{L}$ which occurs at the start of the fast phase is inconsistent with a limit cycle, and comprises strong evidence that jerk $\mathrm{CN}$ is not intrinsically periodic. Moreover, as the deterministic refixating fast phase modelled in section 6.5 predicts periodicity, the local dimension calculations indicate that the fast 
phase is more dynamically complex than a homoclinic reinjection.

\section{Implications of the time series analysis}

\subsection{Jerk nystagmus is caused by a bifurcation in the oculomotor com- mand system}

It is currently believed, on the basis of control models of the oculomotor system, that CN either results from an unstable neural integrator (Optican and Zee, 1984; Tusa et al., 1992), or from an instability of the smooth pursuit system (Harris, 1995; Dell'Osso, 2002; Jacobs and Dell'Osso, 2004).

By proposing a generalised model of the unforced oculomotor system and relating it to recorded jerk nystagmus time series using nonlinear dynamics techniques, evidence has been found supporting the hypothesis that the initial loss of stability in jerk CN is caused by a bifurcation at a fixed point corresponding to stable gaze at the primary position. Furthermore, by matching the experimentally measured eigenvalues of linearisation at this fixed point with those predicted by the model, and comparing with the results obtained from simulated data produced by the unstable NI model of Optican et al ((Optican and Zee, 1984)), it has been deduced that the bifurcation is unlikely to have it's origin in the NI. The loss of stability appears instead to be induced by a bifurcation in one of the five oculomotor subsystems, referred to collectively in the oculomotor system model as the oculomotor command system. Moreover, since the bifurcation is 1-dimensional, it cannot be the direct cause of jerk CN, but is likely to be a preliminary instability preceding oscillatory behaviour caused by a secondary bifurcation (e.g. a Hopf bifurcation). The NI cannot be the cause of this secondary bifurcation either, as the equation for the NI (3) is 1-dimensional, and thus any bifurcation associated with the NI must be 1-dimensional also. These conclusions are in agreement with a previous study that also rejected the NI as the cause of jerk CN, on the basis of estimates of the NI time constant from experimental data (Harris, 1995).

Since the OCS represents the combined dynamics of the oculomotor subsystems, the primary and secondary bifurcations leading to $\mathrm{CN}$ oscillations could, in principle, be caused by parameter changes in any of the individual components. Indeed, there appears to be no a priori reason to favour a particular system, such as smooth pursuit, as the origin of the bifurcations, purely on the basis of the morphology of the resulting time series. This point has been further illustrated by a recent nonlinear dynamics model of the saccadic system which is able to generate $\mathrm{CN}$ waveforms with both slow and fast phases, despite having no slow eye movement components (Broomhead et al., 2000). A range of CN waveforms can be simulated by the model by varying parameters representing the strength of the saccadic braking pulse and the reaction time of saccadic burst neurons to the motor error signal that drives their firing (Broomhead et al., 2000; Akman et al., 2005). In the model, a fixed point 
representing stable gaze at the primary position loses stability in a pitchfork bifurcation as the braking strength is increased, producing a pair of stable fixed points corresponding to hypometric saccades. As the braking strength is increased still further, the fixed points undergo Hopf bifurcations, leading to a pair of limit cycle attractors corresponding to leftbeating and right-beating jerk oscillations (Akman et al., 2005). This picture is consistent with the proposition that the jerk instability is caused by a 1-dimensional bifurcation at a fixed point, followed by a secondary bifurcation which generates an oscillation.

\subsection{The fast phase in jerk $\mathrm{CN}$ is not a simple deterministic refixation}

Current models of jerk CN based on both control theory ((Optican and Zee, 1984; Tusa et al., 1992; Dell'Osso, 2002; Jacobs and Dell'Osso, 2004)) and nonlinear dynamics ((Broomhead et al., 2000; Akman et al., 2005)) produce fast phases that are a deterministic refixation of the target. As detailed in section 6.5, an analytical return map can be derived by combining the simplest model of such a fast phase - a near-homoclinic orbit - with a model of the slow phase dynamics obtained from the data. The latter is derived using the estimates of the eigenvalues of linearisation at the fixed point $\overline{\mathbf{z}}$ representing stationary fixation at primary. Analysis of the return map implies that the attractor of the system should be a stable limit cycle. Consequently, the local dimension $d_{L}$ of the attractor should decrease towards 1 as the cycle moves away from the foveation position. By contrast, $d_{L}$ was found to increase from the value 3 at foveation to 4 just prior to the fast phase. The increase in $d_{L}$ comprises strong evidence against a limit cycle, and suggests a genuinely aperiodic system. This finding is consistent with a previous study which found evidence that the jerk CN attractor may contain several unstable periodic orbits (Clement et al., 2002c). Moreover, it implies that for a model to fully account for the data, it must result in a fast phase with greater complexity than a reinjection of the flow into the neighbourhood of $\overline{\mathbf{z}}$, induced by a near-homoclinic orbit.

\subsection{Signal-dependent noise may account for the nonperiodic nature of jerk CN}

The local dimension estimates suggest that the jerk $\mathrm{CN}$ attractor $\mathcal{A}$ is more complex than the simplest oscillatory mechanism; a stable limit cycle. One possible explanation of the observed variation in $d_{L}$ consistent with the implication of a higher-dimensional attractor is that the dimension $m$ of the manifold $M$ containing $\mathcal{A}$ is greater than or equal to 4 , with contracting directions causing $m$ to be under-estimated in certain regions of $\mathcal{A}$. It follows that additional oculomotor components must be activated as the cycle enters the fast phase, leading to a higher-dimensional refixation mechanism than a homoclinic reinjection.

Alternatively, the variation in local dimension may indicate the presence of a signaldependent noise process. Experimental recordings of human motor unit activity show that the standard deviation of motoneuron firing increases with the mean level of activity 
(Clammam, 1969; Matthews, 1996). In a recent study, Harris and Wolpert incorporated this observation into a control model of the saccadic system by modelling the saccadic velocity command $b_{S}(t)$ as the sum of a deterministic signal $u_{S}(t)$ and a zero-mean, white noise process $w_{S}(t)$ with variance proportional to $\left|u_{S}(t)\right|^{2}$ (Harris and Wolpert, 1998). They found that the mean firing profiles for $u_{S}(t)$ which minimised post-saccadic positional variance produced saccades with position and velocity profiles very similar to those observed experimentally. Harris and Wolpert concluded from this that signal-dependent noise is a significant component of the saccadic system (Harris and Wolpert, 1998). As the firing of the saccadic burst neurons is maximal just prior to a saccade (van Gisbergen et al., 1981), such noise might account for the increase in local dimension at the start of the fast phase, and the subsequent aperiodicity of the waveform. Moreover, it would result in a refixation which was stochastic, consistent with the finding that aperiodicity is incompatible with a low-dimensional refixation.

\subsection{Nonlinear time series analysis can be used to test and develop im- proved oculomotor models}

The eigenvalue and local dimension estimates reported here can be viewed in the context of a growing number of nonlinear dynamics techniques currently being employed to complement control theory in developing biologically realistic models of the oculomotor system (Shelhamer, 1997; Clement et al., 2002a,b,c; Akman et al., 2005). Bifurcation analysis of such models enables predictions to be made regarding which particular behaviours should be observed when system parameters are manipulated experimentally, and in which order the transitions between behaviours will be observed (Wilson, 1999; Akman et al., 2005). Nonlinear time series analysis can assist in the validation and development of the models by providing quantitative techniques for assessing whether the predicted bifurcations occur experimentally. Moreover, time series analysis can uncover new bifurcations, which realistic models must be able to reproduce.

Following this approach, a comprehensive jerk CN model should be able to reproduce the eigenvalue estimates and variations in local dimension reported here. In particular, all current models of jerk CN generate periodic oscillations, and thus do not fully reflect the observed behaviour (Optican and Zee, 1984; Broomhead et al., 2000; Jacobs and Dell'Osso, 2004). The dimension estimates suggest that incorporating the signal-dependent noise characteristic of human motoneurons may lead to more realistic models. For example, using the results of Harris and Wolpert, the saccadic model proposed in (Broomhead et al., 2000) could be modified by adding a white noise process with variance proportional to the mean firing rate of the saccadic burst neurons to the velocity command. The importance of signal-dependent noise as a contributing factor to the jerk mechanism could then be assessed by computing $d_{L}$ for time series generated by the model. If it was found that $d_{L}$ was not significantly affected by the inclusion of signal-dependent noise, it would be 
reasonable to infer that the dimension variation was due instead to the recruitment of additional oculomotor components prior to the fast phase. This hypothesis could be tested in turn by calculating the local dimension for eye movements simulated by a model which included a broader range of oculomotor subsystems, such as smooth pursuit.

Acknowledgements: The authors are grateful to Jerry Huke, Mark Muldoon and Davide Marenduzzo for useful discussions. They also thank the referees for their careful reading of the manuscript and useful comments. O.E. Akman was supported by a grant from the Engineering and Physical Sciences Research Council.

\section{References}

Abadi RV, Bjerre A (2002) Motor and sensory characteristics of infantile nystagmus. Br. J. Ophthalmol. 86: 1152-1160.

Abadi RV, Broomhead DS, Clement RA, Whittle JP, Worfolk R (1997) Dynamical systems analysis: A new method of analysing congenital nystagmus waveforms. Exp. Brain Res. 117: $355-361$.

Abadi RV, Dickinson CM (1986) Waveform characteristics in congenital nystagmus. Doc. Ophthalmol. 64: 153-167.

Abadi RV, Dickinson CM, Pascal E, Whittle J, Worfolk R (1991) Sensory and motor aspects of congenital nystagmus. In: R Schmid and D Zambarbieri, eds. Oculomotor Control and Cognitive Processes. Elsevier Science Publishers BV, The Netherlands. pp. 249-262.

Abadi RV, Sandikcioglu M (1975) Visual resolution in congenital pendular nystagmus. Am. J. Optom. Phys. Opt. 52: 573-581.

Abadi RV, Worfolk R (1989) Retinal slip velocities in congenital nystagmus. Vision Res. 29(2): 195-205.

Akman OE (2000) Nonlinear time series analysis of congenital nystagmus waveforms. Technical report, Mathematics Department, University of Manchester Institute of Science and Technology.

Akman OE (2003) Analysis of a Nonlinear Dynamics Model of the Saccadic System. PhD thesis, Department of Mathematics, University of Manchester Institute of Science and Technology.

Akman OE, Broomhead DS, Abadi RV, Clement RA (2005) Eye movement instabilities and nystagmus can be predicted by a nonlinear dynamics model of the saccadic system. J. Math. Biol. In press. Published online 6th Jun 2005. DOI: 10.1007/s00285-005-0336-4. 
Alligood KT, Sauer TD, Yorke JA (1996) Chaos: An Introduction to Dynamical Systems. Springer-Verlag, New York.

Barnett S (1990) Matrices: Methods and Applications. Clarendon Press, Oxford.

Becker W, Klein HM (1973) Accuracy of saccadic eye movements and maintenance of eccentric eye positions in the dark. Vision Res. 13: 1021-1034.

Bedell HE, Loshin DS (1991) Interrelations between measures of visual acuity and parameters of eye movement in congenital nystagmus. Invest. Ophth. Vis. Sci. 32: 416-421.

Broomhead DS, Clement RA, Muldoon MR, Whittle JP, Scallan C, Abadi RV (2000) Modelling of congenital nystagmus waveforms produced by saccadic system abnormalities. Biol. Cybern. 82: 391-399.

Broomhead DS, Jones R (1989) Time series analysis. P. Roy. Soc. Lond. A 423: 103-121.

Broomhead DS, Jones R, King GP (1987) Topological dimension and local coordinates from experimental data. J. Phys. A 20: L563-L569.

Broomhead DS, King GP (1985) Extracting qualitative dynamics from experimental data. Physica D 20: 217-236.

Broomhead DS, King GP (1986) On the qualitative analysis of experimental dynamical systems. In: S Sarkar, ed. Nonlinear Phenomena and Chaos. Adam Hilger, Bristol. pp. $113-144$.

Cannon SC, Robinson DA (1987) Loss of the neural integrator of the oculomotor system from brain stem lesions in monkey. J. Neurophysiol. 57(5): 1383-409.

Carpenter RHS (1988) Movements of the Eyes. Pion, London.

Clammam PH (1969) Statistical analysis of motor unit firing patterns in human skeletal muscle. Biophysics J. 9: 1233-1251.

Clement RA, Abadi RV, Broomhead DS, Whittle JP (2002a) A new framework for investigating both normal and abnormal eye movements. Prog. Brain Res. 140: 499-505.

Clement RA, Abadi RV, Broomhead DS, Whittle JP (2002b) Periodic forcing of congenital nystagmus. In: S Boccaletti, BJ Gluckman, J Kurths, LM Pecora and ML Spano, eds. Experimental Chaos: Proceedings of the 6th Experimental Chaos Conference, Potsdam, Germany, July 2001. AIP. pp. 149-154.

Clement RA, Whittle JP, Muldoon MR, Abadi RV, Broomhead DS, Akman O (2002c) Characterisation of congenital nystagmus waveforms in terms of periodic orbits. Vision Res. 42: 2123-2130. 
Cohen B, Komatsuzaki A (1972) Eye movements induced by stimulation of the pontine reticular formation: Evidence for integration in oculomotor pathways. Exp. Neurol. 36 (1): 101-17.

Dell'Osso LF (2002) Nystagmus basics: Normal models that simulate dysfunction. In: GK Hung and KJ Ciuffreda, eds. Models of the Visual System. Kluwer Academic, New York. pp. 711-739.

Dell'Osso LF, Daroff MD (1975) Congenital nystagmus waveforms and foveation strategy. Doc. Ophthalmol. 39: 155-182.

Ditchburn RW (1973) Eye Movements and Visual Perception. Clarendon Press, Oxford.

Gancarz G, Grossberg G (1998) A neural model of the saccade generator in the reticular formation. Neural Networks 11: 1159-1174.

Gibson J, Farmer JD, Casdagli M, Eubank S (1992) An analytic approach to practical state space reconstruction. Physica D 57: 1-30.

Glendinning P (1994) Stability, Instability and Chaos. Cambridge University Press, Cambridge.

Goldman MS, Kaneko CRS, Major G, Aksay E, Tank DW, Seung HS (2001) Linear regression of eye velocity on eye position and head velocity suggests a common oculomotor neural integrator. J. Neurophysiol. 88: 659-665.

Goldstein HP (1987) Modeling post-saccadic drift: Dynamic overshoot may be passive. In: Proceedings of 13th Annual Northeast Bioengineering Conference, Philadelphia, USA, March 1987. pp. 245-248.

Guckenheimer J, Holmes P (1983) Nonlinear Oscillations, Dynamical Systems and Bifurcations of Vector Fields. Springer-Verlag, New York.

Harris CM (1995) Problems in modelling congenital nystagmus: Towards a new model. In: JM Findlay, R Walker, and RW Kentridge, eds. Eye Movement Research: Mechanisms, Processes and Applications. Elsevier, Amsterdam. pp. 239-253.

Harris CM, Wolpert DM (1998) Signal-dependent noise determines motor planning. Nature 394: $780-784$.

Harrison PK, Tattersall JEH, Clement RA (2004) Periodic orbit analysis reveals subtle effects of atropine on epileptiform activity in the guinea-pig hippocampal slice. Neurosci. Lett. 357(11): 183-6.

Healey JJ, Broomhead DS, Cliffe KA, Jones R, Mullin T (1991) The origins of chaos in a modified van der Pol oscillator. Physica D 48: 322-339. 
Huke JP (1993) Embedding nonlinear dynamical systems, a guide to Takens' theorem. Internal report, DRA Malvern.

Hundley D, Kirby M (2003) Estimation of Topological Dimension. In: D Barbara and C Kamath, eds. Proceedings of the Third SIAM International Conference on Data Mining, San Francisco, USA, May 2003. SIAM. pp. 194-202.

Jacobs JB, Dell'Osso LF (2004) Congenital nystagmus: Hypothesis for its genesis and complex waveforms within a behavioral ocular motor system model. J. Vision 4: 604625 .

Kantz H, Schreiber T (1997) Nonlinear Time Series Analysis. Cambridge University Press, Cambridge.

Kirby M (2000) Geometric Data Analysis: An Empirical Approach to Dimensionality Reduction and the Study of Patterns. Wiley \& Sons, New York.

Leigh RJ, Zee DS (1999) The Neurology of Eye Movements. Oxford University Press, Oxford.

Matthews PBC (1996) Relationship of firing intervals of human motor units to the trajectory of post-spike after-hyperpolarization and synaptic noise. J. Physiol. 492: 597-628.

Optican LM, Zee DS (1984) A hypothetical explanation of congenital nystagmus. Biol. Cybern. 50: 119-134.

Ott E, Sauer T, Yorke J (1994) Coping with Chaos. Wiley, New York.

Press WH, Teukolsky SA, Vetterling WT, Flannery BP (1992) Numerical Recipes in C: The Art of Scientific Computing. Cambridge University Press, Cambridge.

Robinson DA (1964) The mechanics of human saccadic eye movements. J. Physiol. 174: $245-264$.

Robinson DA (1968) Eye movement control in primates. Science 161(3847): 1219-1224.

Robinson DA (1986) Is the oculomotor system a cartoon of motor control? Prog. Brain Res. 64: 411-417.

Shelhamer M (1997) On the correlation dimension of optokinetic nystagmus eye movements: Computational parameters, filtering, nonstationarity and surrogate data. Biol. Cybern. 76: 237-250.

Stark J (1999) Delay embeddings of forced systems: I. deterministic forcing. J. Nonlinear Sci. 9(3): 255-332. 
Takens F (1981) Detecting strange attractors in turbulence. In: DA Rand and LS Young, eds. Lecture Notes in Mathematics, vol. 898. Springer-Verlag, Berlin. pp. 366-381.

Tusa RJ, Zee DS, Hain TC, Simonsz HJ (1992) Voluntary control of congenital nystagmus. Clin. Vision Sci. 7: 195-210.

van Gisbergen JAM, Robinson DA, Gielen S (1981) A quantitative analysis of generation of saccadic eye movements by burst neurons. J. Neurophysiol. 45: 417-442.

Westheimer G, McKee SP (1975) Visual acuity in the presence of retinal image motion. J. Opt. Soc. Am. 65: 847-850.

Wilson HR (1999) Spikes, Decisions \& Actions: Dynamical Foundations of Neuroscience. Oxford University Press, Oxford.

Zee DS, Yamazaki A, Butler PH, Gucer G (1981) Effects of ablation of flocculus and paraflocculus of eye movements in primate. J. Neurophysiol. 46(4): 878-99. 


\begin{tabular}{|c||c|c|}
\hline & Subject A & Subject B \\
\hline \hline$\hat{\lambda}_{1}$ & $40.9921 \pm 9.1294(0.1136)$ & $20.2361 \pm 5.4983(0.1386)$ \\
\hline$\hat{\lambda}_{2}$ & $-0.0320 \pm 13.1070(208.9715)$ & $-1.3009 \pm 4.1209(1.6161)$ \\
\hline$\hat{\lambda}_{3}$ & $-290.5783 \pm 28.4076(0.0499)$ & $-226.6948 \pm 50.4033(0.1134)$ \\
\hline$E_{F}$ & 0.0629 & 0.0349 \\
\hline$E_{T}$ & 0.0745 & 0.0367 \\
\hline
\end{tabular}

Table 1: Final estimates of the observable eigenvalues $\lambda_{i}$ of $D_{\mathbf{z}} \mathbf{F}(\overline{\mathbf{z}})$ obtained from the experimental data. Errors correspond to the $95 \%$ confidence intervals calculated from the bootstrap samples. The normalised standard errors $\hat{\lambda}_{i}^{E}$ of the estimates are shown in brackets. $E_{F}$ and $E_{T}$ denote the RMS error of the least-squares fit $\Lambda_{L S}$ to the map $\Lambda$ for the fit and test vectors respectively.

\begin{tabular}{|c||c|}
\hline$\hat{\eta}_{1}$ & $15.0415 \pm 0.4373(0.0148)$ \\
\hline$\hat{\eta}_{2}$ & $-48.5301 \pm 1.5326(0.0161)$ \\
\hline$E_{F}$ & 0.0144 \\
\hline$E_{T}$ & 0.0145 \\
\hline
\end{tabular}

Table 2: Final estimates of the eigenvalues $\eta_{i}$ of the matrix $\ln \left(\Lambda^{\frac{1}{\tau}}\right)$ for the simulated jerk $\mathrm{CN}$ data generated by the unstable NI model of Optican et al. Errors correspond to the 95\% confidence intervals calculated from the bootstrap samples. The normalised standard errors $\hat{\eta}_{i}^{E}$ of the estimates are given in brackets. $E_{F}$ and $E_{T}$ denote the RMS error of the least-squares fit $\Lambda_{L S}$ to the map $\Lambda$ for the fit and test vectors respectively. 


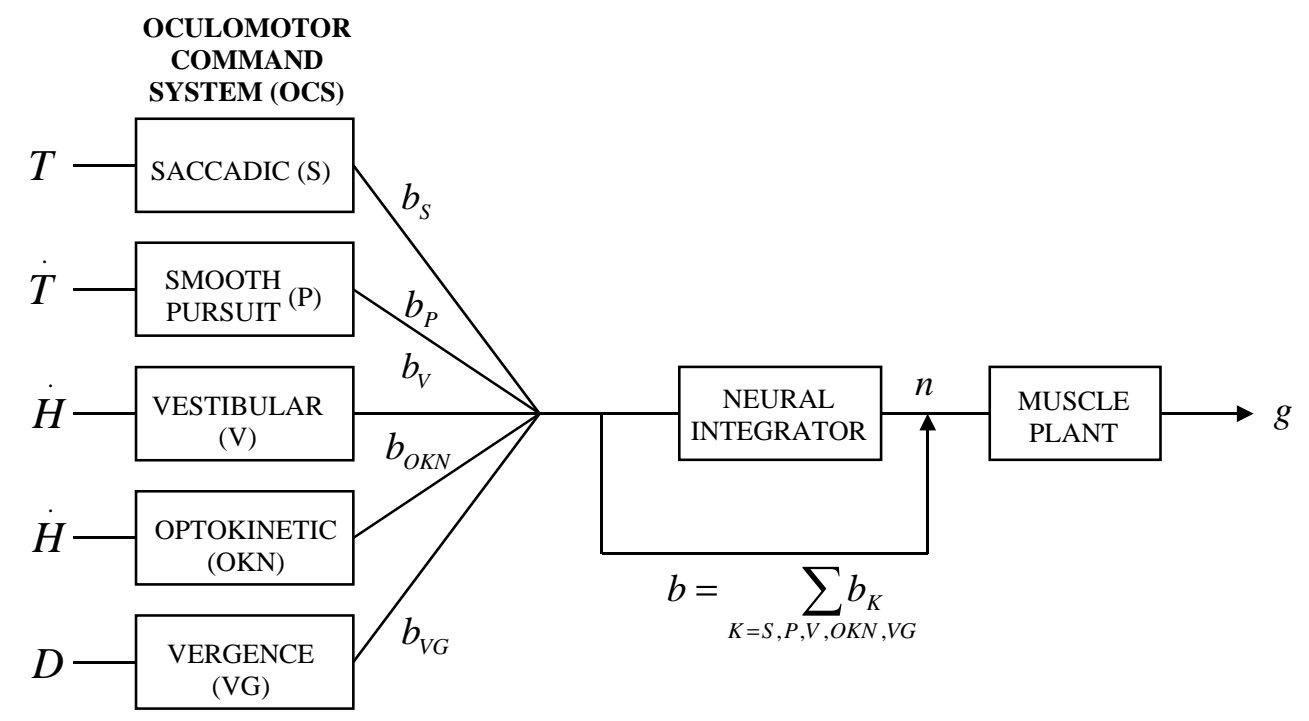

Figure 1: Functional organisation of the oculomotor system for horizontal eye movements. The saccadic system is driven by target position $T$ while the smooth pursuit system responds to target velocity $\dot{T}$. The vestibular and optokinetic systems are driven by a head velocity signal $\dot{H}$ provided by the semicircular canals and visual system respectively. The vergence system is driven by the distance to the target $D . b_{K}=$ velocity signal from subsystem $K, b=$ velocity signal from the OCS, $n=$ position signal from the neural integrator, $g=$ horizontal eye position. (Modified from figure 12.2 of (Carpenter, 1988)).
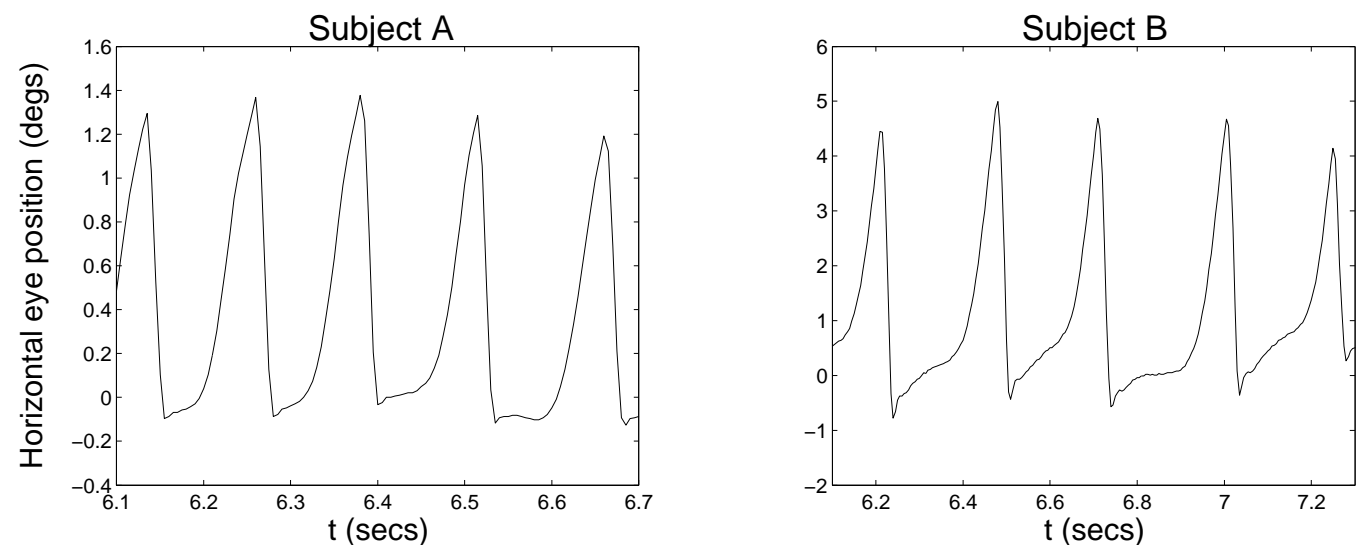

Figure 2: Portions of eye movement recordings obtained from two subjects (A and B) with jerk nystagmus. $0^{\circ}$ indicates gaze straight ahead (primary position). Positive values represent rightward movements. 

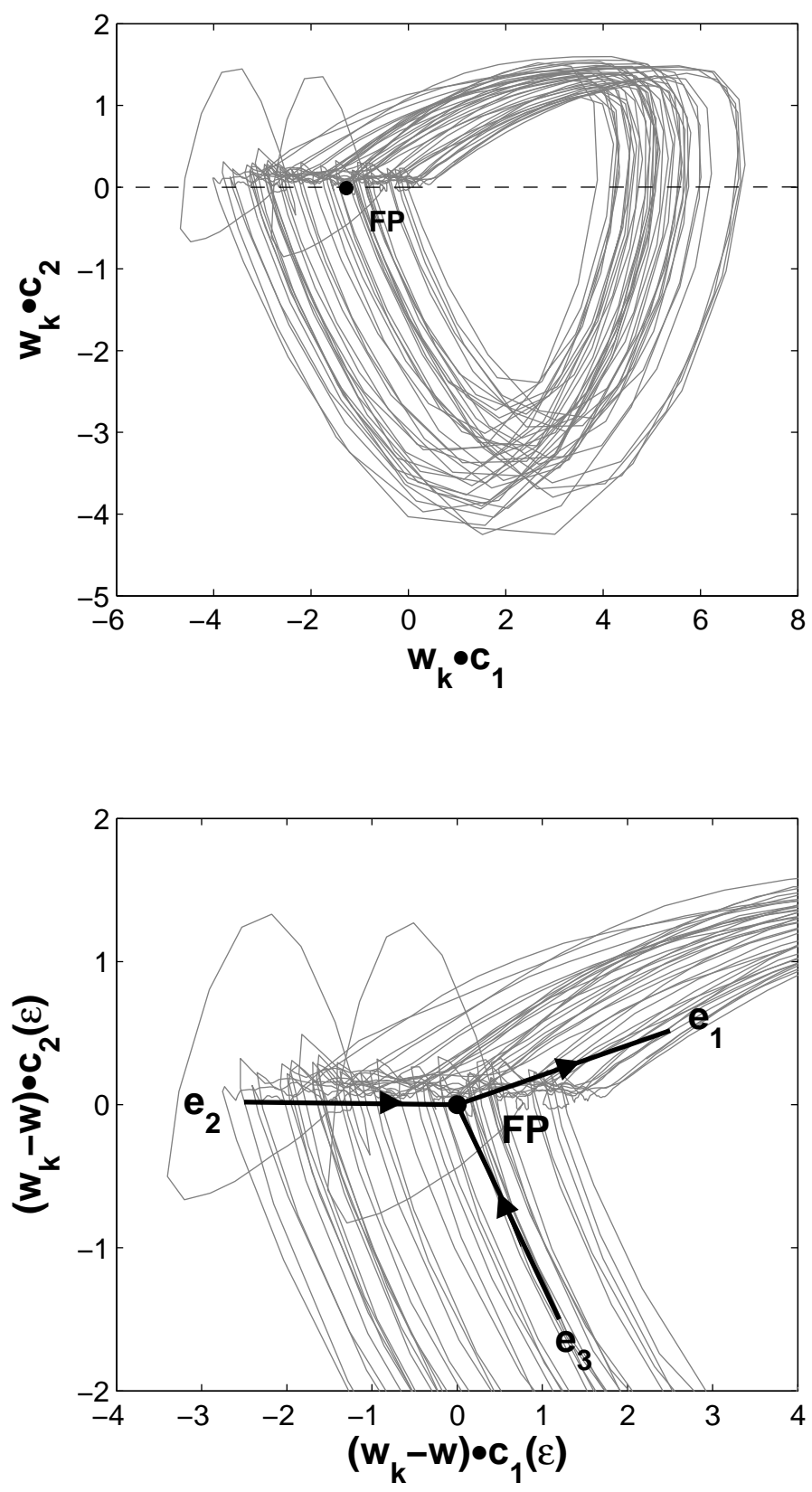

Figure 3: Global and local dynamics reconstructed from the time series of subject B using the method of delays. Upper panel. Global dynamics. Projections of the delay vectors $\mathbf{w}_{k}$ onto the first two singular vectors $\left\{\mathbf{c}_{1}, \mathbf{c}_{2}\right\}$ of the global trajectory matrix $X$. FP represents the estimate $\mathbf{w}$ of the hypothetical fixed point $\overline{\mathbf{w}}$, while the dotted line is the projection of the principal diagonal of the delay space onto $\mathbf{c}_{1}$ and $\mathbf{c}_{2}$. Lower panel. Dynamics in the neighbourhood of the fixed point estimate. Projections of $\mathbf{w}_{k}-\mathbf{w}$ onto the first two singular vectors $\left\{\mathbf{c}_{1}(\varepsilon), \mathbf{c}_{2}(\varepsilon)\right\}$ of the local trajectory matrix $X_{\varepsilon}(\mathbf{w})$, for the choice of ball radius $\varepsilon$ used to obtain the final estimate $\Lambda_{L S}$ of the linear map $\Lambda$ governing the dynamics in the SVD basis. $\left\{\mathbf{e}_{1}, \mathbf{e}_{2}, \mathbf{e}_{3}\right\}$ represent the eigenvectors of $\Lambda_{L S}$; the arrows indicate the direction of the flow along the eigenvectors. 

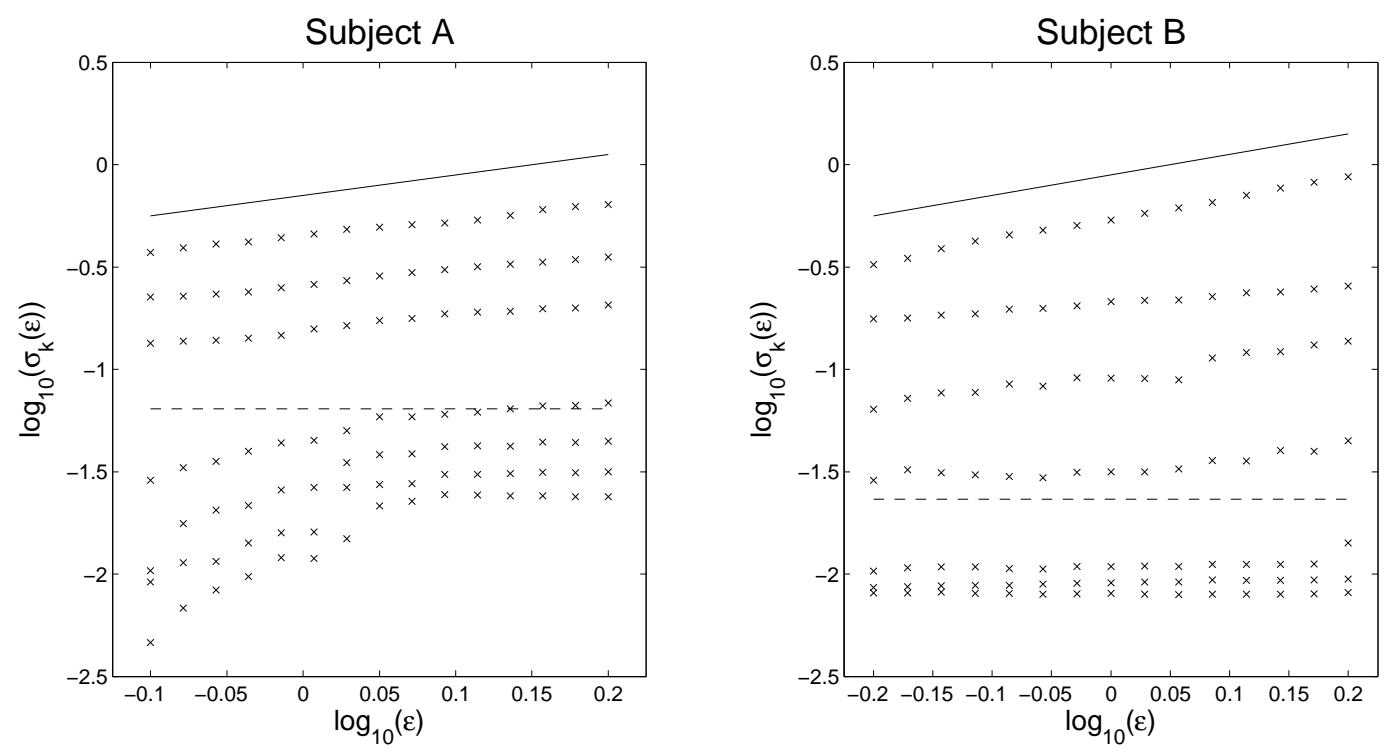

Figure 4: Scaling of local singular values at the estimate of the hypothetical fixed point for the experimental data sets. In each plot, the dotted line indicates the noise floor $\sigma_{n}$, defined as the ratio of the measurement device resolution to the standard deviation of the unnormalised time series. The solid line represents the orientation at which the singular values scale linearly with $\varepsilon$. 

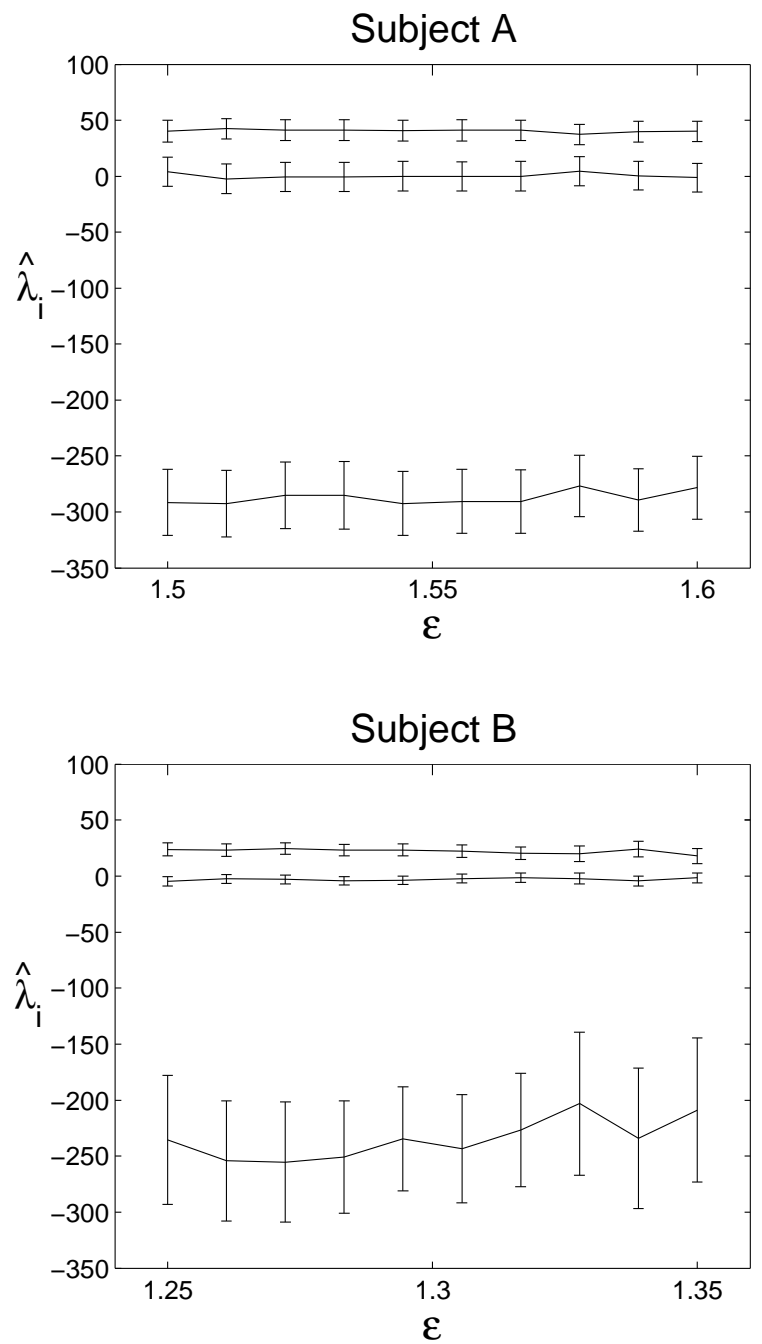

Figure 5: Estimates $\hat{\lambda}_{i}$ of the observable eigenvalues $\lambda_{i}$ of $D_{\mathbf{z}} \mathbf{F}(\overline{\mathbf{z}})$, obtained from the experimental time series using the method of section 6.3. Each plot shows the variation of $\hat{\lambda}_{i}$ with the size $\varepsilon$ of the ball containing the delay vectors used to compute the leastsquares fit $\Lambda_{L S}$ to the map $\Lambda$. In each plot, the top, middle and bottom lines represent $\hat{\lambda}_{1}$, $\hat{\lambda}_{2}$ and $\hat{\lambda}_{3}$ respectively. Error bars indicate the $95 \%$ confidence intervals, calculated using the bootstrap technique described in section 6.4. 


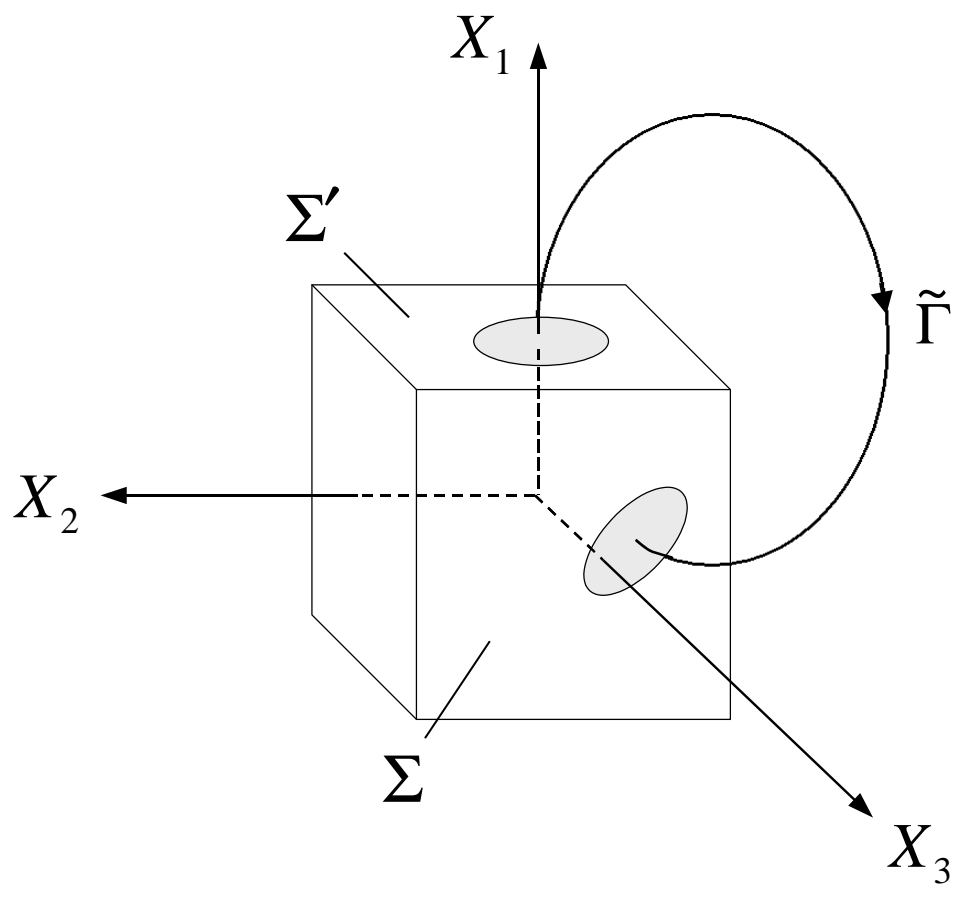

Figure 6: Construction of a return map for the unforced oculomotor system model $\dot{\mathbf{z}}=\mathbf{F}(\mathbf{z})$, derived from the experimental data. The origin corresponds to a fixed point $\overline{\mathbf{z}}$, representing stationary gaze at the primary position, which has undergone a bifurcation leading to the jerk instability. Within the cube, the dynamics are approximately linear and represent the motion of the eye close to foveation. The dynamics outside the cube are assumed to be a reinjection mechanism induced by a near-homoclinic orbit $\tilde{\Gamma}$, modelling a refixating fast phase. A return map $\mathbf{P}$ to the surface of section $\Sigma$ is obtained by composing two maps $\mathbf{P}_{1}$ and $\mathbf{P}_{2}$, where $\mathbf{P}_{1}$ - constructed from the flow inside the cube - maps from $\Sigma$ to $\Sigma^{\prime}$, and $\mathbf{P}_{2}$ - constructed from the flow outside the cube - maps from $\Sigma^{\prime}$ to $\Sigma$. 

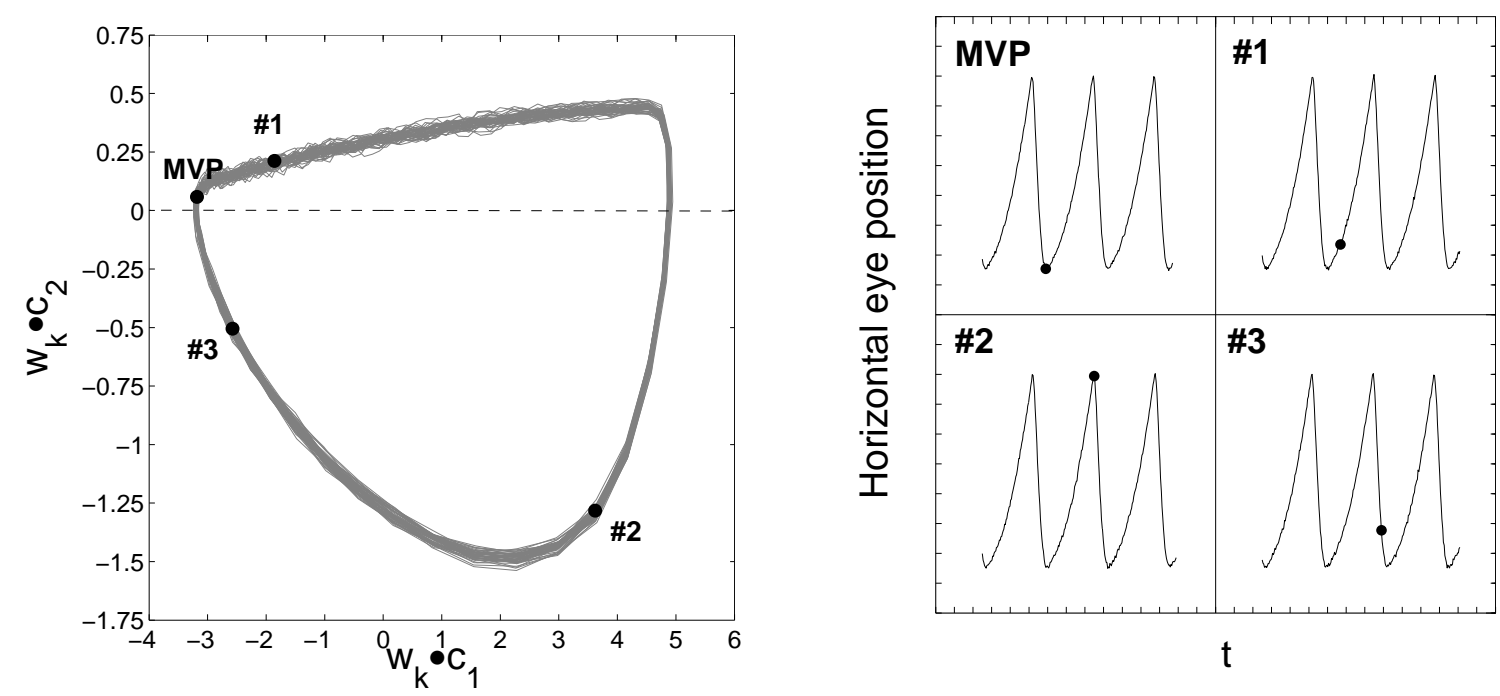

Figure 7: Left panel. Projection of the delay vectors $\mathbf{w}_{k}$ for the unstable NI model data onto the first two singular vectors $\left\{\mathbf{c}_{1}, \mathbf{c}_{2}\right\}$ of the global trajectory matrix $X$. MVP denotes the delay vector obtained by applying the fixed point estimation technique of section 6.1 to the $\mathbf{w}_{k} \mathrm{~s}$, while $\# 1, \# 2$ and $\# 3$ denote the points in the CN cycle at which $d_{L}$ was computed to investigate the local dimension changes consistent with a limit cycle. The dotted line is the projection of the principal diagonal of the delay space onto $\mathbf{c}_{1}$ and $\mathbf{c}_{2}$. Right panel. The time series values corresponding to the points at which $d_{L}$ was calculated. The tick marks on the horizontal axis represent $100 \mathrm{~ms}$ intervals while those on the vertical axis denote intervals of $0.5^{\circ}$. 

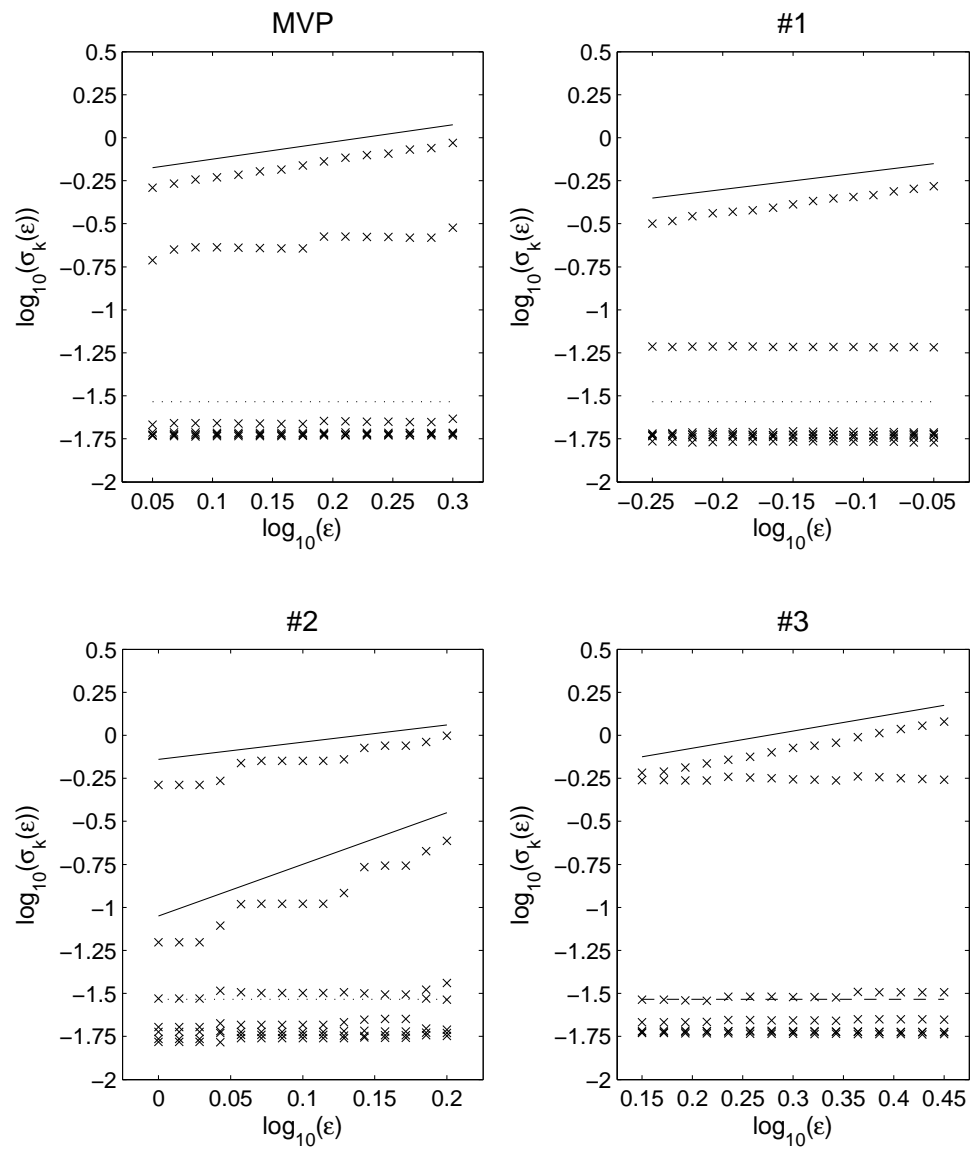

Figure 8: Local singular value scaling at points MVP, \#1, \#2 and \#3 of figure 7 . In each plot, the dotted line indicates the noise floor $\sigma_{n}$. For points MVP, \#1 and \#3, the solid line shows the orientation at which the singular values scale linearly with the ball radius $\varepsilon$. For point $\# 2$, the upper solid line represents a scaling exponent of 1 . The lower solid line represents a scaling exponent of 3 , for which the corresponding singular value represents the curvature of the attractor orthogonal to the principal singular vector. 

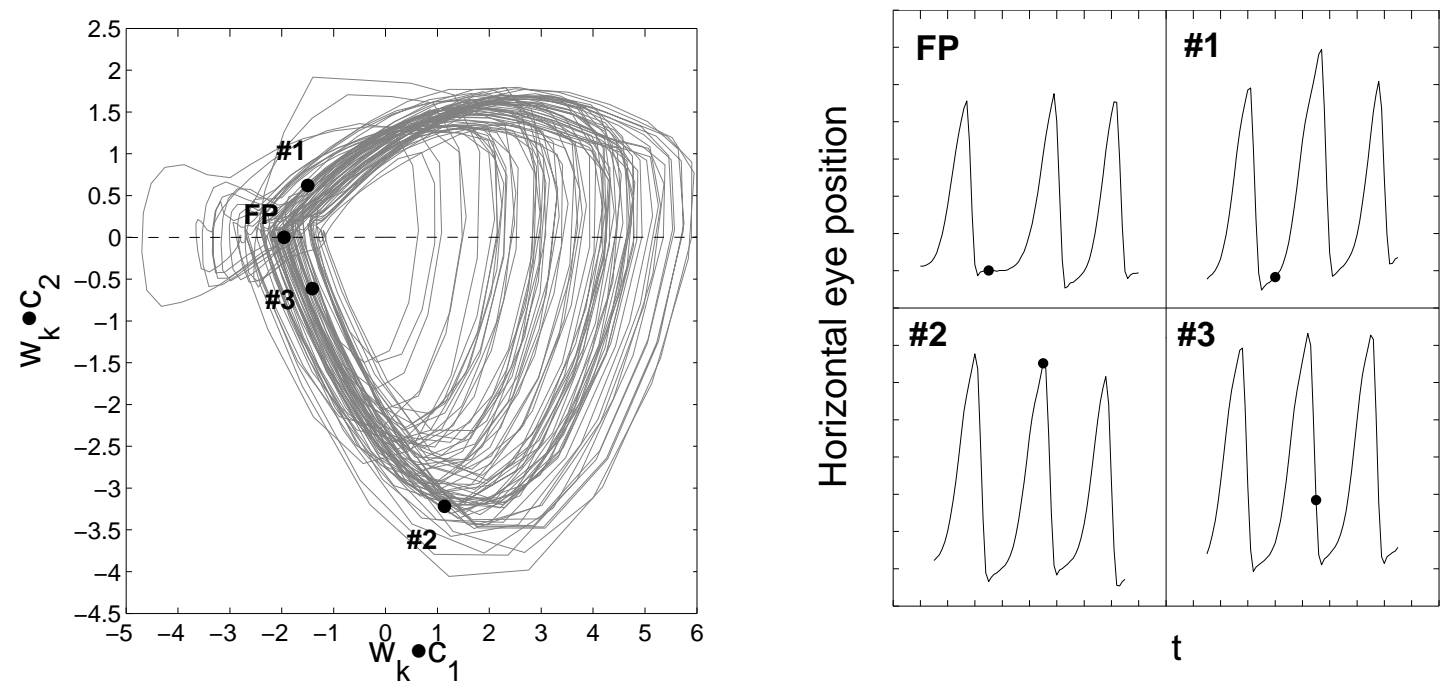

Figure 9: Left panel. Projection of the delay vectors $\mathbf{w}_{k}$ for subject A onto the first two singular vectors $\left\{\mathbf{c}_{1}, \mathbf{c}_{2}\right\}$ of the global trajectory matrix $X$. FP denotes the estimate of the fixed point $\overline{\mathbf{w}}$, while $\# 1, \# 2$ and $\# 3$ denote the points in the $\mathrm{CN}$ cycle at which $d_{L}$ was computed to assess if $\operatorname{dim}(M)=1$. The dotted line is the projection of the principal diagonal of the delay space onto $\mathbf{c}_{1}$ and $\mathbf{c}_{2}$. Right panel. The time series values corresponding to the points at which the local dimension was calculated. The tick marks on the horizontal axis represent $50 \mathrm{~ms}$ intervals while those on the vertical axis denote intervals of $0.25^{\circ}$.
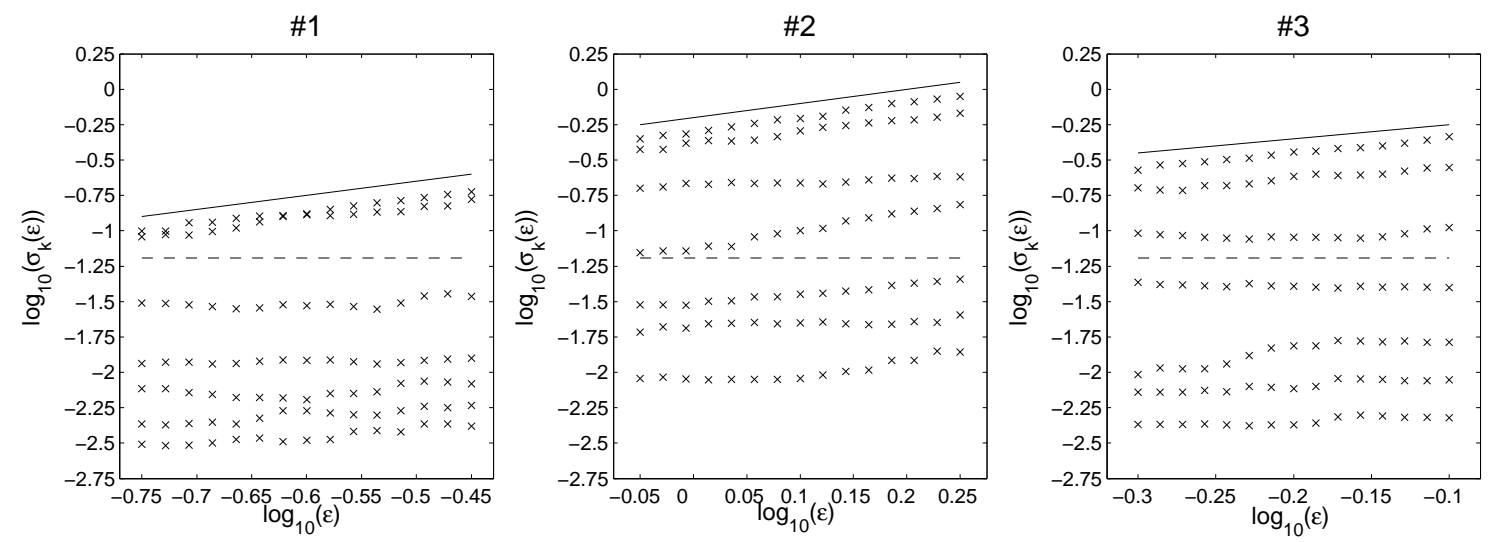

Figure 10: Local singular value scaling at points \#1, \#2 and \#3 of figure 9. In each plot, the dotted line indicates the noise floor $\sigma_{n}$, while the solid line shows the orientation at which the singular values scale linearly with the ball radius $\varepsilon$. 